\title{
Design of experiment and analysis techniques for fuel consumption data using heavy-duty diesel vehicles and on-road testing
}

Sarah Ann Mills

West Virginia University, samills@mix.wvu.edu

Follow this and additional works at: https://researchrepository.wvu.edu/etd

Part of the Other Mechanical Engineering Commons, Other Statistics and Probability Commons, and the Statistical Methodology Commons

\section{Recommended Citation}

Mills, Sarah Ann, "Design of experiment and analysis techniques for fuel consumption data using heavyduty diesel vehicles and on-road testing" (2019). Graduate Theses, Dissertations, and Problem Reports. 3815.

https://researchrepository.wvu.edu/etd/3815

This Thesis is protected by copyright and/or related rights. It has been brought to you by the The Research Repository @ WVU with permission from the rights-holder(s). You are free to use this Thesis in any way that is permitted by the copyright and related rights legislation that applies to your use. For other uses you must obtain permission from the rights-holder(s) directly, unless additional rights are indicated by a Creative Commons license in the record and/ or on the work itself. This Thesis has been accepted for inclusion in WVU Graduate Theses, Dissertations, and Problem Reports collection by an authorized administrator of The Research Repository @ WVU. For more information, please contact researchrepository@mail.wvu.edu. 


\title{
Design of experiment and analysis techniques for fuel consumption data using heavy-duty diesel vehicles and on- road testing
}

\author{
Sarah A. Mills
}

Thesis submitted to the

Benjamin M. Statler College of Engineering and Mineral Resources at West Virginia University

in partial fulfillment of the requirements

for the degree of

Master of Science in

Mechanical Engineering

Cosmin Dumitrescu, Ph.D., Chair

V'yacheslav Akkerman, Ph.D.

Arvind Thiruvengadam, Ph.D.

Department of Mechanical and Aerospace Engineering

West Virginia University

Morgantown, West Virginia

2019

Keywords: on-road data collection, fuel consumption, big data Copyright 2019 Sarah Mills 


\title{
ABSTRACT \\ Design of experiment and analysis techniques for fuel consumption data using heavy-duty diesel vehicles and on-road testing
}

\author{
Sarah A. Mills
}

Chassis dynamometer and on-road testing are usually employed to test vehicle operation. Testing on a chassis dynamometer reduces data variability compared to on-road testing due to the controlled environment but it does not account for other important variables that affects real-world vehicle operation. This study used on-road testing to investigate the differences between two test fuels under real-world conditions. Three heavy-duty diesel vehicles were driven on different routes for a period of three months. Each vehicle was instrumented with flow meters to gather fuel consumption data, which was then compared to the fuel rate broadcasted by the engine control unit (ECU). Additionally, the driveshaft torque was measured using a strain gage and a torque transmitter, which was used to confirm that the output torque was correlated to the vehicle's fuel consumption. Data from both the ECU and the sensors were stored on a portable activity measurement system (PAMS), which also collected global positioning system (GPS) data and ambient conditions. The experimental procedure was based on SAE J1321. Due to the proprietary nature of the data, specific results of the study were not shown. However, the thesis details the design of experiments, including the selection, installation, benefits, and limitations of using additional sensors to improve data analysis. It also discusses the data storage and methods used for data analysis with the considerably large data sets obtained in the study. For example, while $\sim 4.5$ million data points were collected for each vehicle and each month of testing, more than $55 \%$ of the data points were discarded due to idling, engine cutoff during downhill operation, and adverse weather conditions. With respect to data analysis, the principal component analysis (PCA) identified the variables that caused the most variability in the datasets. PCA and data binning were used to compare datasets and determine the differences between them. The results show that the route with the most interstate data supplied the highest number of usable data points. Moreover, the ECU fuel consumption was consistent with the flow meter data with an average percent error of $2.5 \%$. Measuring the engine torque using a torque meter can be difficult for on-road testing due to the excessive vibration experienced by the sensor. 


\section{Acknowledgements}

I am so thankful for all the people in my life that have helped make this thesis possible.

I am eternally grateful to my parents, Robert and Katrina Mills, for their constant love and support throughout my life and academic career. Thank you for being my biggest role models and for teaching me the power of hard work and determination. Your achievements are inspirational, and I feel so blessed to have you as my parents. I would also like to thank Jared Dotson for his unwavering love and support throughout this journey to attain my Master's degree. I am so fortunate to have you.

Thank you to Dr. Cosmin Dumitrescu for serving as my advisor for the past year and a half. Thank you for teaching me more than I could have ever imagined during my time as a Master's student. Thank you for entrusting me to manage the project and for your support and help in completing this thesis. Also, thank you to Dr. V'yacheslav Akkerman and Dr. Arvind Thiruvengadam for agreeing to be my committee members and for taking the time to review this thesis.

Thank you to all the members of CAFEE at the Vehicle and Engine Testing Laboratory for their friendship and their assistance in instrumenting the vehicles. I would not have been able to complete this project without their knowledge and support.

I would like to sincerely thank Grover, Roger, and Tony for their dedication to driving the vehicles throughout the duration of the study. They took great pride in their work and were always going above and beyond for the success of the experiment. I especially appreciate their help with maintaining the operation of the sensors on a daily basis, which was not an easy task.

Finally, thank you to all the incredible people I've met through West Virginia University that have made this experience great. These people include Amber, Brian, Dakota, Rasik, Renata, Sam (both) and so many more. 


\section{Table of Contents}

Acknowledgements ...................................................................................................................................... iii

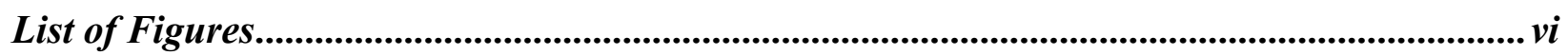

List of Tables............................................................................................................................................. vii

List of Abbreviations..................................................................................................................................... viii

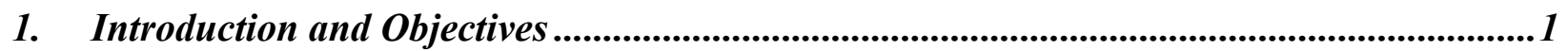

$1.1 \quad$ Introduction ..................................................................................................................................1

$1.2 \quad$ Objectives ...................................................................................................................................

2. Literature Review..................................................................................................................

2.1 Procedures to Measure Fuel Consumption..................................................................................3

2.1.1 Dynamometer Fuel Consumption Testing........................................................................................

2.1.2 SAE J1321 Standard for On-Road Fuel Consumption Testing .......................................................

2.2 Factors that Influence Fuel Consumption ..................................................................................7

2.2.1 Effects of Engine Operation on Fuel Consumption ………………………………………………..... 8

2.2.2 Effects of Route Selection on Fuel Consumption ………………………………………………….....9

2.2.3 Effects of Time of Day on Fuel Consumption ............................................................................10

2.2.4 Effects of Driver Behavior on Fuel Consumption..............................................................................11

2.2.5 Effects of Vehicle Components on Fuel Consumption …………………………………………... 12

2.3 Strategies for Analyzing Big Data...........................................................................................13

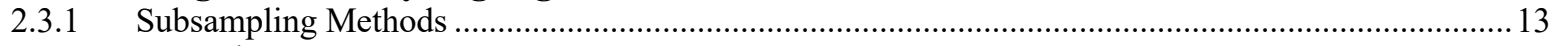

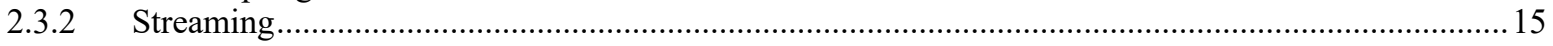

2.4 Principal Component Analysis.................................................................................16

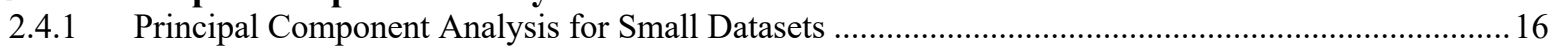

2.4.2 Principal Component Analysis for Big Data ………………………………………………….... 19

3. Experimental Setup ....................................................................................................22

3.1 Experimental Methods..........................................................................................................22

3.2 Vehicle Details ............................................................................................................................23

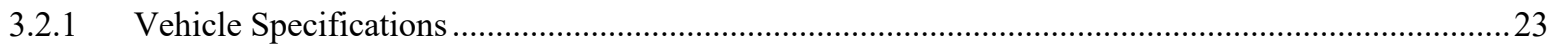

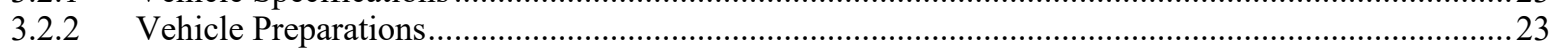

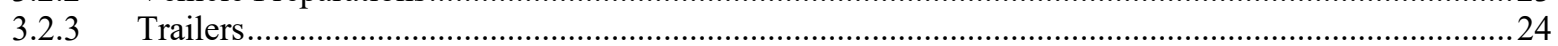

$3.3 \quad$ Routes ................................................................................................................................25

$3.4 \quad$ Vehicle Operator Daily Tasks .................................................................................................27

3.5 Fuel Flow Meters..............................................................................................................................28

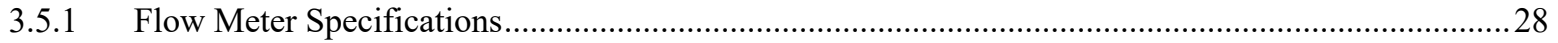

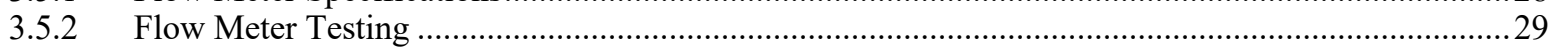

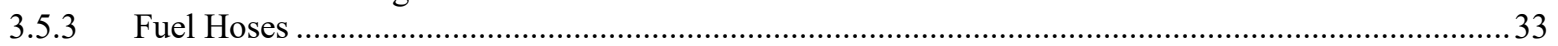

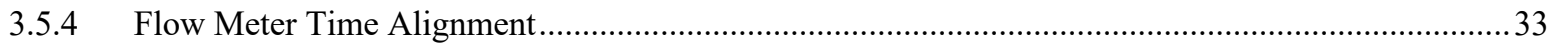

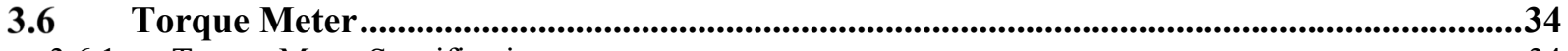

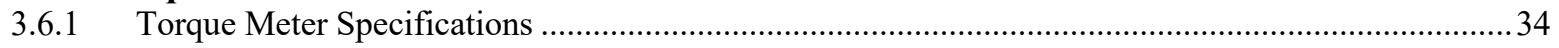

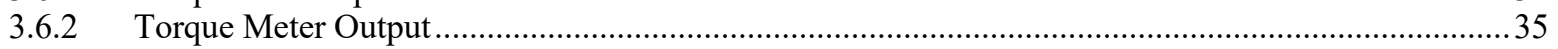

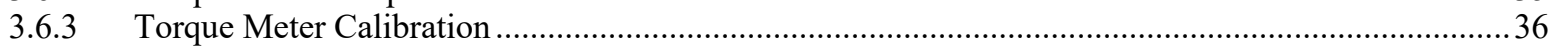

3.6.4 Strain Gage ……………………………………………………………………………… 37

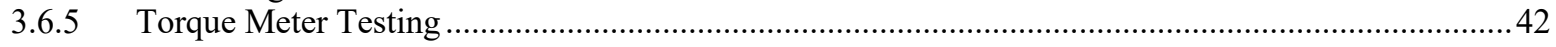




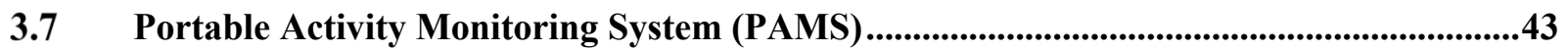

3.8 Printed Circuit (PC) Board for Data Acquisition ...............................................................43

4. Data Collection and Analysis..................................................................................46

4.1 Data Collection and Storage .......................................................................................................46

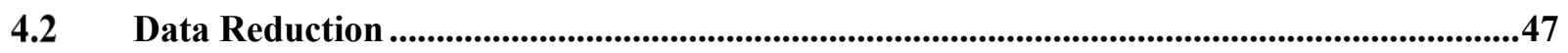

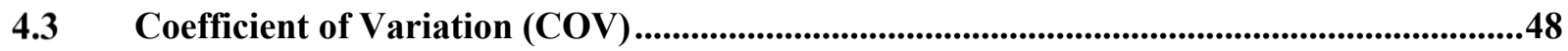

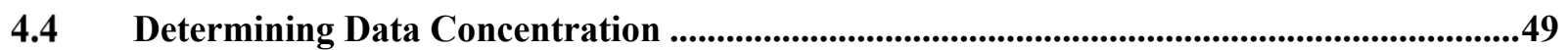

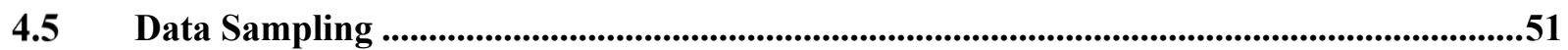

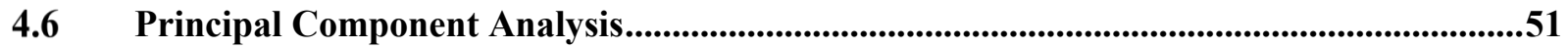

4.7 Binning by Additional Variables ..................................................................................................52

4.8 Comparing Data Sets ....................................................................................................................55

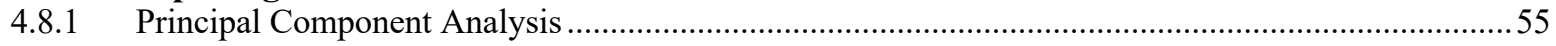

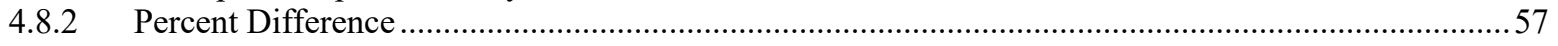

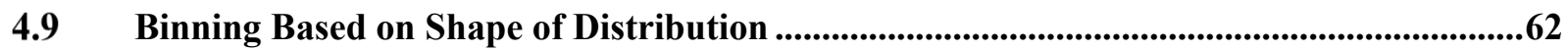

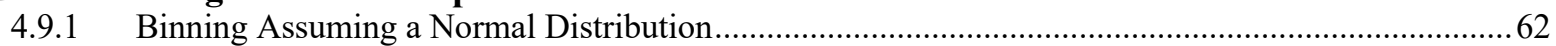

4.9.2 Binning Accounting for Shape of Distribution .................................................................................6 63

5. Summary, Conclusions, and Recommendations for Future Work ...................................70

$5.1 \quad$ Summary and Conclusions ...................................................................................................................70

5.2 Recommendations for Future Work .........................................................................................74

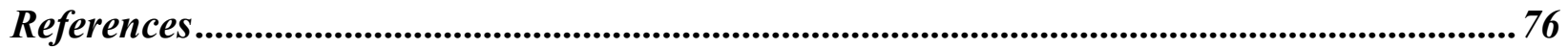




\section{List of Figures}

Figure 2.2.1: Principal Component Separation Example [28] ....................................................17

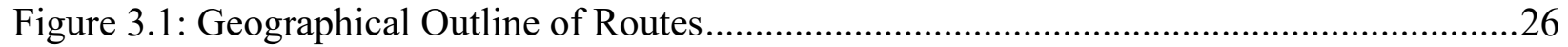

Figure 3.2: Percent Difference of ECU and KRAL Fuel Consumption for Each Mode of SET

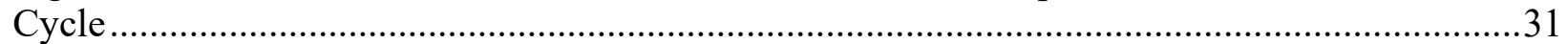

Figure 3.3: Comparison of ECU and KRAL Flow Rate for Varying Engine Power .....................32

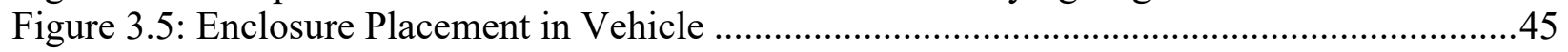

Figure 4.1: Example COV Contour Plot .............................................................................49

Figure 4.2: Number of Data Points Contour Plot Example .............................................................50

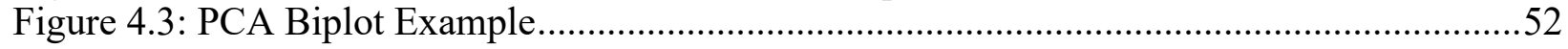

Figure 4.4: Visualization of Binned Matlab Structure …………………….............................55

Figure 4.5: Principal Component Comparison Without Separation .............................................57

Figure 4.6: Positive Percent Difference Histogram Example........................................................59

Figure 4.7: Negative Percent Difference Histogram Example ……………………........................59

Figure 4.8: Zero Percent Difference Histogram Example ............................................................6

Figure 4.9: Low Kurtosis and Negative Skewness Example...........................................................65

Figure 4.10: High Kurtosis and Positive Skewness Example ………………………….................65

Figure 4.11: Example of Range Optimization for Skewness .......................................................68 


\section{List of Tables}

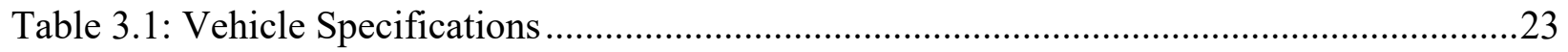

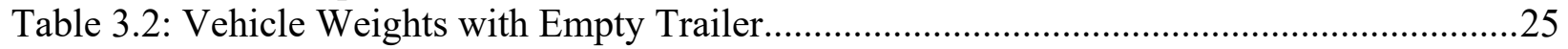

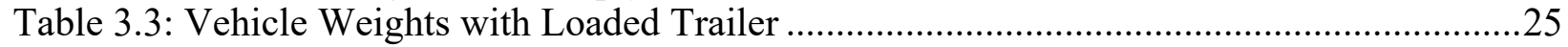

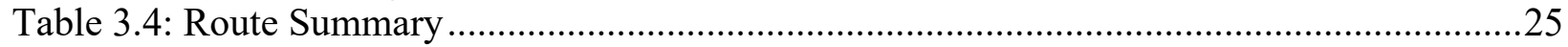

Table 3.5: Percentage of Data Points for Each Route Type ...................................................27

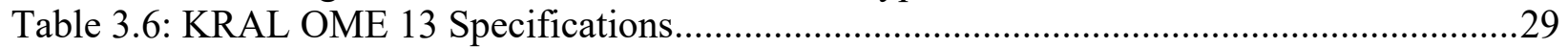

Table 3.7: Summary of SET Cycle Modes .......................................................................... 30

Table 3.8: Percent Difference Between Fuel Consumption According to KRAL and ECU.........33

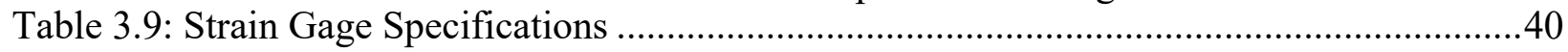

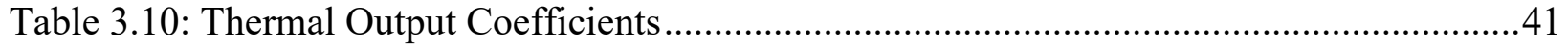

Table 4.1: Properties of the Distribution in Figure 4.11 ..........................................................6 


\section{List of Abbreviations}

$\begin{array}{ll}\text { AEE } & \text { Aggregated Estimating Equation } \\ \text { AMT } & \text { Automated Manual Transmission } \\ \text { bsfc } & \text { Brake-Specific Fuel Consumption } \\ \text { CAFEE } & \text { Center for Alternate Fuels Engines and Emissions } \\ \text { CAN } & \text { Controller Area Network } \\ \text { CO } & \text { Carbon Monoxide } \\ \text { CO }_{2} & \text { Carbon Dioxide } \\ \text { COV } & \text { Coefficient of Variation } \\ \text { DPF } & \text { Diesel Particulate Filter } \\ \text { ECU } & \text { Engine Control Unit } \\ \text { EGR } & \text { Exhaust Gas Recirculation } \\ \text { FTP } & \text { Federal Test Procedure } \\ \text { GPS } & \text { Global Positioning System } \\ \text { HC } & \text { Hydrocarbons } \\ \text { HDDV } & \text { Heavy Duty Diesel Vehicle } \\ \text { HP } & \text { Horsepower } \\ \text { ID } & \text { Inner Diameter } \\ \text { IMT } & \text { Intake Manifold Temperature } \\ \text { OD } & \text { Outer Diameter } \\ \text { PAMS } & \text { Portable Activity Monitoring System } \\ \text { PC } & \text { Printed Circuit } \\ \text { PCA } & \text { Principal Component Analysis } \\ \text { PeMS } & \text { Performance Measurement System } \\ \text { PM } & \text { Particulate Matter } \\ \text { SET } & \text { Supplemental Emissions Test } \\ \text { SAE } & \text { Society of Automotive Engineers } \\ \text { SD } & \text { Standard Deviation } \\ \text { SVD } & \text { Singular Value Decomposition } \\ \text { VETL } & \text { Vehicle and Engine Testing Laboratory } \\ \text { WV } & \text { West Virginia } \\ & \end{array}$




\section{Introduction and Objectives}

\subsection{Introduction}

Heightened online shopping is increasing freight delivery inside cities [1]. As the fuel is a major component of the freight-delivery cost to the companies, any improvement in fuel economy will result in major cost benefits over the lifetimes of the delivery truck [2]. Moreover, fuel consumption is directly proportional to carbon dioxide $\left(\mathrm{CO}_{2}\right)$ emissions [3]. According to the Environmental Protection Agency (EPA), transportation (which includes both heavy-duty vehicles and passenger cars) contributed $28 \%$ of the total greenhouse gas emissions in 2016 [4]. There is motivation to reduce the $\mathrm{CO}_{2}$ emissions to keep the $\mathrm{CO}_{2}$ concentration at or below 450 ppm by 2050 in order to "help to put the planet onto a longer term path to environmental sustainability [5]." An $80 \%$ decrease in $\mathrm{CO}_{2}$ emissions from the $1990 \mathrm{CO}_{2}$ level is the goal to achieve by 2050 $[5]$.

\subsection{Objectives}

Hellstrom et al. [2] found that just a few-percent improvement in fuel consumption can cause a substantial decrease in the cost to use the vehicle over its lifetime. The objective of this work was to develop a method for capturing differences in on-road engine operation (fuel consumption, efficiency, emissions, etc.) for vehicles operating using two different fuels. This objective was achieved by:

- Operating three heavy-duty diesel vehicles (1 control vehicle and 2 test vehicles) on specified routes for a period of 3 months 
- Collecting and storing data from parameters broadcasted by the engine control unit (ECU) at a frequency of $10 \mathrm{~Hz}$

- Utilizing flow meters to measure fuel consumption and a torque meter to measure torque on the driveshaft

- Collecting GPS and ambient condition data from a portable activity monitoring system (PAMS)

- Completing statistical analysis to compare datasets from each segment of testing to determine differences in engine parameters for each fuel 


\section{Literature Review}

\subsection{Procedures to Measure Fuel Consumption}

Fuel consumption can be measured in a laboratory using a dynamometer or on-road. Each measuring method has its own advantages and disadvantages.

\subsubsection{Dynamometer Fuel Consumption Testing}

Two types of dynamometers are generally used for fuel consumption testing: engine dynamometers and chassis dynamometers. Chassis dynamometer testing involves operating a vehicle. As the wheels are rotating on the rollers of a chassis dynamometer, the vehicle can operate normally without moving. Engine dynamometers connect directly to the output shaft of an engine that has been removed from a vehicle. Regulatory emissions testing is completed using either an engine or chassis dynamometer, depending of the certification, using standard engine cycles. One such standard engine cycle is the Federal Test Procedure (FTP) cycle, which involves operating the engine for different combinations of transient activities [6]. This is done to ensure that collected data can be used to compare the engine to a standard.

Engine dynamometer testing is useful for regulations but may not be effective for fuel consumption testing because this type of testing does not account for accessories such as air conditioning or for transmission efficiency. Air conditioning in an actual vehicle can affect fuel consumption by a maximum of $20 \%$ [7]. Therefore, chassis dynamometer testing gives a better idea about fuel consumption compared to an engine dynamometer.

Chassis dynamometer testing is advantageous compared to on-road testing because many variables can be held constant, so there is higher experimental repeatability and control $[8,9]$. 
However, according to Tong et al [10], only testing engines in a laboratory may not give accurate results when trying to make conclusions for on-road vehicles. Chassis dynamometer testing uses a fan to cool the engine and simulate the air flowing around the vehicle during operation [11]. Therefore, the fuel consumption measurements using this testing does not account for varying wind speed and direction. Moreover, while chassis dynamometer testing neglects the effect of road conditions on the rolling resistance, a chassis dynamometer can simulate different rolling resistances by changing the tire type and/or pressure [11].

\subsubsection{SAE J1321 Standard for On-Road Fuel Consumption Testing}

On-road testing involves instrumenting a vehicle with sensors and recording vehicle operation data while travelling on a road. As on-road testing introduces many more variables than chassis dynamometer testing, it uses real world conditions to determine actual differences in fuel consumption whenever and wherever a vehicle is driven. The Society of Automotive Engineers (SAE) developed a test procedure (SAE J1321) for on-road testing designed to gather fuel consumption data from vehicles under real conditions [12]. The procedure is very detailed and offers specific instructions in order to minimize variability in the data. The following sections discuss the rules for completing a fuel consumption test according to SAE J1321.

\subsubsection{Test Vehicles}

According to SAE J1321 the test vehicles should have similar (preferably the same) external surface contours to ensure a consistent aerodynamic drag force across all vehicles. Therefore, it is best to procure the same make, model, and model year vehicles for testing. There should be at least two vehicles used for the duration of testing, where one is used as the control 
vehicle, so no modifications will ever be made during testing. There must also be at least one test vehicle that is modified (e.g., another fuel) for a portion of the testing. The tires on all vehicles should be in similar condition, preferably with the same mileage accumulated on each tire. New tires should not be used, and each tire should have been driven for at least 500 miles, so that the results of the study will be applicable to existing fleets with used tires. The vehicles should all have similar mileages, especially if the mileage on each truck is less than thirty-thousand miles. SAE J1321 outlines specific mileage constraints for these cases. However, the difference in mileage between the trucks is negligible if all of the trucks have accumulated more than thirtythousand miles prior to testing [12].

The trailers, like the vehicles, must have the same external surface contours and each trailer must be assigned to a specific vehicle for the entire length of testing if adding load to a vehicle is desired. Additionally, the weight of each vehicle should be representative of the fleet for which the test is being modeled. In other words, if the test is being used to determine differences in fuel economy for a fleet that typically carries 20,000 pounds of material each day, then 20,000 pounds should be added to the trailer. The added weight should be solid because liquid loads will shift and introduce inconsistencies in the load experienced by each axle. Also, the load should not change throughout the testing period unless it is desired to determine differences in fuel consumption at different loads. According to the procedure, the total weight of each vehicle and trailer should be the same, but if that is not achievable, the difference in weight should be less than $1.5 \%$ [12].

\subsubsection{Drivers}

All drivers should have extensive heavy-duty vehicle driving experience in order to drive consistently and reduce driver variability between test runs. SAE J1321 states that all drivers should collaborate prior to the beginning of testing to determine where brakes should be applied, 
where gears should be shifted, and where the trucks should accelerate or decelerate throughout the route in order to reduce driver variability. Additionally, all drivers should be interviewed at the end of each test run to document any issues or observations that may have been gathered during the test run [12].

\subsubsection{Instrumentation}

All vehicles must be instrumented with sensors that gather data in addition to the data coming from the engine control unit (ECU). Each vehicle must be instrumented with a GPS unit to record the latitude and longitude of the vehicle and the vehicle speed with a precision of $0.1 \mathrm{mph}$. Each vehicle must also be instrumented with a weather monitoring unit to gather ambient conditions such as air temperature, humidity, barometric pressure, and wind speed.

Finally, external devices for fuel consumption measurement must be installed on the vehicles. The preferred method mentioned in the procedure is to install a gravimetric fuel measurement device. In other words, a device that weighs the fuel tanks should be used for fuel consumption measurement. However, fuel flow meters can be used instead of gravimetric devices if they can effectively measure the fuel consumption with an accuracy of $\pm 0.5 \%$, which should be verified through testing [12].

The strict accuracy requirement for the flow meters is necessary because flow meters can be inaccurate during some areas of operation. During a study by Burke et al [13], they found that the flow meters used to measure fuel consumption have poor repeatabilioty during stop-and-go operation compared to other fuel measurement sensors. They also found that the fuel consumption values from the flow meters had the highest repeatability during the high-power engine operation. Therefore, if the data is segmented based on the power, the high-power results have a higher precision compared to analyzing data at a lower power [13]. 


\subsubsection{Test Run Procedure}

The test is run in two segments: the baseline segment and the test segment. During the baseline segment, all vehicles must complete at least 3 runs without any modifications to determine how the trucks perform before any modifications are made. On the other hand, the control vehicle remains unmodified during the test segment, while the modification is imposed on the test vehicles and at least 3 runs are completed. Each run must be at least 100 miles in length. Utilizing a track for the test runs is recommended, but roads may be used with a specified route if grade change does not exceed 3.5\%. The specified route should begin and end the same approximate location. The vehicles should remain at least 1500 feet away from each other during the runs to minimize aerodynamic interference and differences in ambient conditions between the vehicles. Additionally, testing should not be conducted if rain is present [12].

All the vehicles should be inspected at the end of each testing day to ensure no other changes occurred that could affect vehicle operation, therefore affecting the test results [12].

\subsection{Factors that Influence Fuel Consumption}

As previously mentioned, a small decrease in fuel consumption will make a significant impact on the total fuel consumed by a vehicle over its lifetime [2]. Because of this, many researchers have investigated the factors that influence fuel consumption such as "travel-related, weather-related, vehicle-related, roadway-related, traffic-related, and driver-related factors" [14]. Therefore, based on these categories, factors such as ambient temperature, humidity, wind speed, route selection, engine size and condition, aftertreatment, operating temperature and many others can influence the amount of fuel consumed by an engine [14]. 


\subsubsection{Effects of Engine Operation on Fuel Consumption}

Burke et al. [17] examined the effects of changes in coolant temperature to NOx emissions and fuel consumption. They performed an experiment using "a modern 4-cylinder, 2-liter, turbocharged, high-pressure common-rail direct-injection diesel engine." The engine was connected to a dynamometer, so the data was not collected from on-road testing. The setup was capable of controlling the coolant temperature at four different coolant temperature settings, ranging from $50^{\circ} \mathrm{C}$ to $98^{\circ} \mathrm{C}$. The exhaust gas recirculation (EGR) flow rate and the injection timing were also adjusted. A design of experiments was used to ensure all combinations of the coolant temperature, injection timing, and EGR flow rate were accounted for in the testing, although some conditions were found to be impossible to achieve. The fuel consumption, in this case, was measured by completing a carbon balance from the emissions data to back-calculate the amount of fuel consumed by the engine.

Burke et al. [17] found that when EGR rates and injection timing were held constant, and coolant temperature was decreased from $70^{\circ} \mathrm{C}$ to $50^{\circ} \mathrm{C}$, the brake specific fuel consumption (bsfc) increased by $3.1 \%$ and when the coolant temperature decreased from $98^{\circ} \mathrm{C}$ to $86^{\circ} \mathrm{C}$, the bsfc increased by $2 \%$. Therefore, fuel consumption increased as coolant temperature decreased for both the high, and the low ranges of coolant temperature. In both coolant temperature regions, oxides of nitrogen (NOx) emissions were reduced, so it was concluded that NOx emissions are inversely related to fuel consumption for this study. However, the results suggested changes in EGR flow rate and injection timing have a much larger effect on the NOx and fuel consumption than the coolant temperature [17]. Since injection timing remains constant on an on-road vehicle, it will not make an impact on fuel consumption in on-road testing. However, the EGR flow rate can be monitored as an input variable contributing to a change in fuel consumption. 
Tong et al. [10] tested 4 vehicles (1 passenger vehicle, 1 van fueled with petrol, 1 van fueled with diesel, and 1 double-decker bus) for emissions and fuel consumption in urban areas in Hong Kong. Each vehicle was driven by the same driver to eliminate driver variability. They used a flow meter in the supply line of the fuel system to measure the fuel supplied to the engine. They segregated the data based on both average vehicle speed and driving mode (i.e. idling, accelerating, decelerating, cruising) and they provided the conditions that must be satisfied for a data point to be classified in one of these modes. In general, all vehicles consumed more fuel and released more emissions as the instantaneous vehicle speed increased because the engine must consume more fuel to maintain a higher vehicle speed. For the modal analysis, the acceleration mode proved to cause the highest fuel consumption for the passenger car, petrol van, and the diesel van, which was to be expected since "the engine needs more fuel to generate enough power to accelerate" [10]. The double-decker bus was not tested for fuel-consumption, only emissions, so there is no fuel consumption data. From their study, they concluded that the instantaneous vehicle speed was the variable that impacted the fuel consumption and emissions values the most [10].

\subsubsection{Effects of Route Selection on Fuel Consumption}

Engine power and corresponding fuel consumption are determined by several factors, which include speed and acceleration requirements, the grade of the road the vehicle will be driving on, the aerodynamic characteristics of the vehicle, and rolling resistance of the tires. Boriboonsomsin et al. [15] investigated the effect of changes in road grade in regard to fuel consumption and thus, carbon dioxide emissions. They wrote that if all variables except road grade remain constant, a consistently hilly route may have the same fuel consumption as a flat route of the same distance, because the high fuel consumption during the uphill sections is offset by the low fuel consumption during the downhill sections. Frey et al. [16] also investigated the effects of 
road grade on fuel consumption and emissions. They found that if the road grade was ignored, the fuel consumption and emissions were underestimated by $16-24 \%$. Therefore, road grade has a significant impact on fuel consumption.

Boriboonsomsin et al. [15] also discussed the methods for determining road grade using data from an experiment. A performance measurement system (PeMS) was used determine the geographic location and the speed of the vehicles. Based on the PeMS data, a comprehensive modal emissions model (CMEM) estimated the fuel consumption and, subsequently, the $\mathrm{CO}_{2}$ emissions. Therefore, PeMS and CMEM determined the most fuel-efficient route to a destination. However, it was not ideal to use a topographical map because it was time-consuming and not always up-to-date. Therefore, the study used two carrier-phase differential Global Positioning System (GPS) receivers to calculate the road grade [15]. One receiver was stationed at the starting location of the vehicle and the second receiver was housed on the vehicle. The receivers determined both the elevation and the GPS location of both the vehicle, and the starting location. The latitude and longitude of each vehicle location were used to determine the distance from the starting location. Then, the elevation and distance values were used to calculate the road grade using an equation in their paper. The vehicle's broadcasted fuel consumption rate was used for the fuel consumption measurement. It was concluded that the fuel economy for a light-duty vehicle on a hilly route was $15 \%$ to $20 \%$ lower compared to a flat route, hence the hypothesis that the difference in fuel economy will be much larger for a heavy-duty vehicle because load on a heavyduty vehicle (i.e. trailer and overall weight of vehicle) is higher than the load on a light-duty vehicle [15].

\subsubsection{Effects of Time of Day on Fuel Consumption}


Frey et al. [16] also studied the effects on fuel consumption for driving a test vehicle at different times of the day. It was determined that when the vehicles travelled during peak traffic hours, both fuel consumption and emissions increased, compared to fuel consumption and emissions during off-peak traffic hours. Therefore, data should be segregated based on time of day to accurately compare data points.

\subsubsection{Effects of Driver Behavior on Fuel Consumption}

An on-road study introduces variability based on driver activity, especially if there are multiple vehicles and drivers. Frey et al. [16] studied the effects of driver variability on fuel consumption. It was suggested that the variability between drivers is due to the "different speed profiles" each driver exhibits [16]. Frey et al. conducted an on-road study and found that when all other variables were held constant, the $\mathrm{CO}_{2}$, hydrocarbon ( $\left.\mathrm{HC}\right)$, $\mathrm{NOx}$ and carbon monoxide (CO) emissions all varied. Since the carbon-based emissions varied, the fuel consumption also varied due to driver variability.

Lee and Son [20] conducted a study on the relationship between driving behavior and fuel consumption by analyzing data on 15 drivers on a $\sim 22$ mile stretch of highway. Many parameters were analyzed in addition to the fuel consumption such as vehicle and engine speeds, steering wheel position, acceleration pedal position, road grade, yaw angle, driver body movements, and driver eye position, among others. They mentioned that other studies concluded that the acceleration pedal position offered the best differentiation between driver behavior and fuel consumption. However, their multiple regression analysis on the data concluded that the standard deviation of the steering wheel angle was more indicative because the more the steering wheel moved, the more the driver was passing other vehicles, which means that the fuel consumption 
was higher. While the standard deviation of the steering wheel angle had the highest correlation to fuel consumption, both the average, and the standard deviation of the acceleration pedal position still had a significant correlation to the fuel consumption. Additionally, the average transmission gear gave a lower, but still significant correlation to fuel consumption in this study.

\subsubsection{Effects of Vehicle Components on Fuel Consumption}

Automated manual transmissions (AMT) are most common in heavy-duty fleets that travel long distances. These transmissions do not employ a clutch or torque converter to switch gears. Instead, AMTs use "engine torque control," which changes gears based on the driving conditions, such as road grade and the driver input via the acceleration pedal [18]. Eckert et al. conducted a study and found that utilizing an AMT reduces fuel consumption because it skips the first 2 gears except when the lowest gears are absolutely necessary. Skipping the first 2 gears reduces fuel consumption because those gears cause the highest load on the engine and require high engine speeds [19].

The condition of the tries on a vehicle can also have an effect on fuel consumption. Brace et al. [11] conducted a study using a vehicle on a chassis dynamometer to determine factors that would influence fuel consumption differences between different testing facilities. It was determined that the tire type and tire pressure had a significant effect on fuel consumption. The study used "fuel saver" tires as a baseline and changed the tires to "low-profile sports" tires and found a $3.6 \%$ increase in fuel consumption when the low-profile tires were used, compared to the fuel saver tires. The effect of oil level on fuel consumption values was also investigated. The vehicle's oil reservoir to the upper dipstick mark, and then 2.5 liters was removed from the reservoir to determine a lower oil level's effect on fuel consumption. The lower oil level showed 
a $2.9 \%$ decrease in fuel consumption compared to the fuel consumption with a full reservoir for this study.

\subsection{Strategies for Analyzing Big Data}

Once all the data is collected from a study, it must be analyzed to derive a conclusion. However, because the amount of data can be exorbitant, research has been conducted specifically to handle these large sets of data. The term "big data" is used to classify data that is large in "volume, intensity, and complexity that exceed the ability of standard software tools to manage and analyze [21]." Big data is generated every day when using the internet and sensors, especially when data is collected from sensors at a high frequency. There are 3 major categories for analyzing big data: subsampling-based methods, divide-and-conquer methods, and methods for online updating for streamed data [21].

\subsubsection{Subsampling Methods}

Wang et al. [21] mentioned several subsampling-based methods to use when handling big data. The "Bags of Little Bootstrap" method involves sampling a predetermined number of subsamples of the same size from the raw data set. Then, each subsample is sampled until these bootstrap samples are the same size as the original data set. The bootstrap samples are then used to gather parameters such as mean, median, etc. and confidence intervals. Finally, all the parameters from each bootstrap sample are averaged to find the parameters and confidence intervals representative of the original population. The "Bag of Little Bootstrap" approach should be used when multiple computers are available and necessary to process the analysis due to the size of the raw dataset because each subsample can be computed on a separate processor [21]. 
Another subsampling-based method is leveraging, which takes subsamples of the data, including the weights of each data point, and then performs computations to calculate any of the necessary parameters (mean, standard deviation, etc.), which are intended to represent the full dataset. The weights are used to ensure the data points that affect the parameters the most are more likely to be included in the samples than data points that affect the parameters the least. This technique is recommended when limited processing power is available since this method only computes parameters for small subsets of the full dataset [21].

The mean log-likelihood approach involves taking subsamples of a dataset of the same size and calculating the Monte Carlo averages and the Kullback-Leibler divergence for each subsample. Monte Carlo simulation is used to analyze data and make predictions based on a known range of values. For Monte Carlo analysis, the user provides a range of possible values of a variable. The simulation then generates a probability distribution and chooses a random value from the distribution and generates a model. This process is repeated as many times as the user would like, which could be on the order of thousands of time. The output of the simulation is a probability distribution of the results of the model to show where the actual result is most likely to lie [22]. Additionally, the Kullback-Leibler divergence is a statistical analysis technique that determines the distance between probability distributions, also known as the divergence [23]. Wang et al. discusses that since the mean log-likelihood method uses subsamples much smaller than the actual size of the data, this method is very useful for big data because it can be processed easily. They mention a simulation study conducted using this method, which concluded that the calculations converge to the appropriate value regardless of the full dataset size[21].

For the typical divide-and-conquer strategy, the full dataset is divided into a certain number of sections, where each section is processed and computed individually, and then all solutions are combined to determine a solution for the entire dataset. The aggregated estimating equation (AEE) 
method is the first divide-and-conquer method Wang et al. discusses. This method performs linear regression on each section of data to find the "least squares estimator" of each section. Then, the weight of each least-squares estimator is found by finding "inverse of the estimated variance matrix" to calculate the weighted average of all the least squares estimators, which is the least squares estimator for the entire dataset. This method performs most accurately when the estimating equations are linear. However, if they are not linear, a Taylor series expansion can be used to provide a "linear approximation of the estimating equations" for each section of data. Then, these approximations can be used in the weighted average calculation for the entire dataset. However, the weight for the linear approximation is calculated differently than the previously mentioned weight. The weight for each section of data "is the slope matrix of the estimating function at the solution" in each section[21].

Lin et al. [24] developed an AEE estimator for large datasets and data streams. The estimator first aggregates, or clusters, the data into equally sized sections. Then, an estimating equation estimate is found by calculating the solution to an estimating equation for each section of data, which included in their paper. Then, the AEE estimator can be found for the entire dataset.

Wang et al. [21] discusses several other divide-and-conquer methods. While all methods have their own unique properties, they all follow the same basic premise of the divide-and-conquer strategies, previously mentioned.

\subsubsection{Streaming}

In some cases of big data, the data is collected in "streams," which requires analysis to be updated as the new streams are made available without having to store all the data and run the analysis on the entire dataset. Wang et al. mentions the works of Schifano et al. [25] where a method was developed for analyzing online data streams using linear regression and estimating 
equations. Schifano et al. describes their exact methods for applying linear models and estimating equations to online data streams in detail [21].

\subsection{Principal Component Analysis}

\subsubsection{Principal Component Analysis for Small Datasets}

When the data being analyzed has many input variables, it is necessary to narrow down the variables to determine the most influential input variables on the output variable before the data is analyzed using one or a combination of the methods provided by Wang et al [21, 26]. Principal component analysis (PCA) is one of the most widely used methods to determine the variables that have the most impact on the output variables [26]. PCA uses a dataset with $m$ variables and calculates $m$ principal components, which are linear combinations of the variables supplied in the original data set. The first principal component captures the most variation of the original data set, while each successive principal component captures less variation than the previous principal component [27].

If most of the variation is captured by the first 2 or 3 principal components, the first 2 or 3 principal components can be plotted against each other for multiple datasets to determine if there is a difference between the datasets. One can conclude there is a difference between datasets if the principal components appear in clusters that are separated, where each cluster is associated with a different dataset. In Figure 2.2.1, the $x$ and $y$ axes indicate the first and second principal components, respectively. Therefore, Figure 2.2.1 shows an example of separation between datasets, where each color signifies a different dataset, because each dataset's principal components are separated from each other. In most cases, PCA is the first analysis technique 
completed, so that more traditional techniques, such as linear regression, can be completed using only a few variables [26].

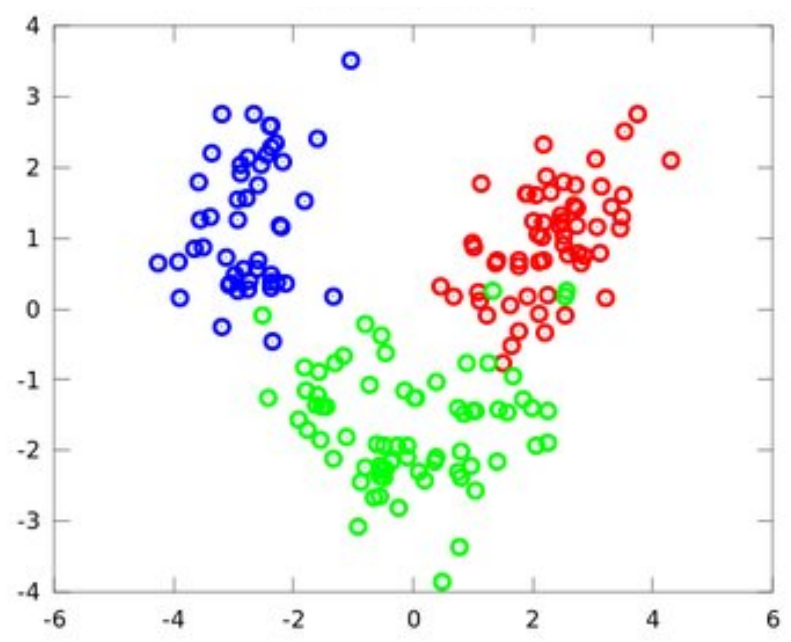

Figure 2.2.1: Principal Component Separation Example [28]

In order to perform PCA on data, the data should be organized in a matrix such that each column represents a different input variable. Also, "the data must be preprocessed" such that "the columns of the data matrix are centered such that the mean of each column is zero [reference]." Additionally, all input variable values should be standardized to ensure all variables are treated equally, since the input variables are not all measured in the same units. In order to standardize the input variable, each column of data should be divided by its "norm," also known as its magnitude. If $\boldsymbol{X}$ is the raw data matrix with dimensions $n \times p$ and $\boldsymbol{X}_{\boldsymbol{s}}$ is the standardized data matrix, each value of $X_{s}$, denoted as $x_{i j, s}$, can be calculated using Equation (1) where $i$ and $j$ indicate the row and column, respectively, of the value of $\mathbf{X}_{\mathbf{s}}$ being calculated from the respective value of $\boldsymbol{X}$ [26]. Therefore, " $\underline{x}_{i j}$ is the $(i, j)$ th entry of $\boldsymbol{X}$ ", $\overline{x_{j}}$ is the mean of the $j$ th column of $\boldsymbol{X}$, and $s_{j}$ "is the sum of squares of errors (SSE) of the $j$ th column" of $\boldsymbol{X}$, which is calculated using Equation (2). 


$$
\begin{gathered}
x_{i j, s}=\frac{x_{i j}-\overline{x_{j}}}{s_{j}} \\
s_{j}=\sqrt{\sum_{i=1}^{n}\left(x_{i j}-\bar{x}_{j}\right)^{2}}
\end{gathered}
$$

Once the data is in this format, singular value decomposition (SVD) can be applied such that $\boldsymbol{X}_{\boldsymbol{s}}$ is decomposed to the form in Equation (3), where $r$ is the rank of $\boldsymbol{X}_{\boldsymbol{s}}$ and $\boldsymbol{X}, \boldsymbol{U}$ "is an $n \times r$ orthogonal matrix" and $\boldsymbol{V}$ "is a $p \times r$ orthogonal matrix", and $\boldsymbol{D}$ "is an $r \times r$ diagonal matrix [26]."

$$
\mathbf{X}_{\mathbf{s}}=\mathbf{U D V}^{\mathrm{T}}
$$

The process for decomposing $\boldsymbol{X}_{\boldsymbol{s}}$ into the form in Equation (3) is detailed by Strang [29]. The diagonals of $\boldsymbol{D}$ are the eigenvalues of $\boldsymbol{X}_{\boldsymbol{s}}{ }^{T} \boldsymbol{X}_{\boldsymbol{s}}$ such that $d_{1} \geq d_{2} \geq \cdots \geq d_{r}>0$. $\mathbf{V}$ is composed of the eigenvectors corresponding to the eigenvalues in $\boldsymbol{D}$, such that the first column on $\boldsymbol{V}$ is the eigenvector for the first value of $\boldsymbol{D}$ and so on. Finally, $\boldsymbol{V}$ is orthogonal, so $\boldsymbol{V}^{T} \boldsymbol{V}=\boldsymbol{I}$ and the relationship in Equation (4) is true [29].

$$
\mathbf{X}_{\mathbf{s}} \mathbf{V}=\mathbf{U D V}^{\mathrm{T}} \mathbf{V}=\mathbf{U D}
$$

Therefore $\boldsymbol{u}_{j}$ can be found using Equation (5), which is a column vector for each value of $j=1 \ldots$ $r$ that make up the matrix $\boldsymbol{U}$. Similarly, $\boldsymbol{v}_{\boldsymbol{j}}$ is the $j$ th column of the matrix $\boldsymbol{V}$ [29].

$$
\mathbf{u}_{\mathrm{j}}=\frac{\mathbf{X}_{\mathbf{s}} \mathbf{v}_{\mathbf{j}}}{\mathrm{d}_{\mathrm{j}}}
$$

After SVD is applied, the principal components are the columns of the matrix $\boldsymbol{U D}$ and the loadings of each principal component are the columns of the matrix $\boldsymbol{V}$ [26]. However, the term 'loading' can have two different meanings. As previously mentioned, the columns of $\boldsymbol{V}$ are referred to as loadings; and, in this case, "the sum of the squared elements of a given component is equal to one [27]." The second meaning of loading varies mostly due to the normalization technique of 
the coefficients. Loading could also be defined as "the correlation between a component and a variable [27]." In this case, the correlation coefficients are normalized such that the sum of each correlation coefficient squared is equal to one [27]. Therefore, as long as the user knows which type of normalization was used, the meaning of "loading" will be clear [27].

Zhang et al. explained the methods for calculating the total variation, $\lambda$, for as many principal components the user would like to check, denoted as $k$. In other words, if the user would like to see how much of the total variation is accounted for each of the first 6 principal components, $k$ would be equal to 6 [26]. Using these methods, the analysis is specifically referred to as "correlation PCA," which is the typical version of PCA used in statistical software that performs PCA [26, 27]. There is another, less common version of PCA, referred to as "covariance PCA." In this case, each of the columns of the raw data matrix are preprocessed such that the "mean of each column is zero [26]." Therefore, the transpose of the data matrix multiplied by the original data matrix is the covariance matrix $[26,27]$.

Several different programs can be used to complete PCA, such as Matlab and JMP. However, these programs are only usable for data that does not exceed the size limitations imposed by the program or computer being used. For example, Matlab is limited by the amount of memory available on the computer being used. Matlab offers extensive explanations and an example called "Analyze Quality of Life in U.S. Cities Using PCA" that can be used to create a Matlab script to complete PCA for a dataset [30].

\subsubsection{Principal Component Analysis for Big Data}

The PCA methods previously mentioned were created for moderately sized data. However, for big data, these methods can be more difficult, if not impossible to implement. The most prevalent issues with big data are both data storage, and speed of computation. For example, SVD 
requires use of the entire data matrix. Therefore, if the matrix is too large to be loaded onto a single computer, PCA cannot be completed without using methods such as an external memory algorithm, where the data is processed using several if not thousands of processors. Similarly, if the data matrix grows continuously because data is being stored in real-time, the matrix is constantly changing and PCA cannot be completed [26].

The correlation PCA approach can be used if the data is small enough to be stored in the memory of a computer. However, if the data matrix is too large to be stored in the memory, but small enough to be stored on a single computer's hard disk, more complicated methods can be used to apply PCA to the data. Zhang et al. [26] developed a log-likelihood function for a linear regression model of the data. The coefficients and matrices inside the log-likelihood function can be obtained using an algorithm as long as a $p \times p$ matrix can be stored in the memory of the computer being used, because that is the largest dimension matrix inside the log-likelihood function. After applying the algorithm, a matrix $\boldsymbol{C}_{x x}$ is found, which has dimensions $p \times p$. This matrix is used to find the principal components. In short, this method uses the raw data matrix that is too large to be stored in the memory of a computer and creates a matrix that is dependent on the number of variables, rather than the number of data entries, which is small enough to be stored in the memory of the computer.

Zhang et al. [26] also describes a procedure for applying PCA to data collected in streams. In this case, a new matrix is derived once new data is added, removed, or both added, and removed. Then, the same algorithm previously mentioned is utilized. Therefore, PCA can be applied to any size of data.

Abdi et al. [27] describes the process for plotting the results of PCA and the significance of the plot. The output of PCA analysis is a coordinate system with the first and second principal components as the horizontal and vertical axes, respectively. The coordinate system will have a 
circle, referred to as the "circle of correlations," which, if the data is perfectly explained by the first two principal components, the loadings will be located on the circle. This is because in order to be a circle, the sum of the squared coordinates of each point is equal to a constant. Therefore, if "the sum of the squared loadings is equal to one," "the data is perfectly represented by only two components [27]." However, if the data is not fully explained by the first two components, the loadings will be located between the circle and the origin, where the closer each variable is to the origin, the less it is influenced by the first two components [27].

Ericsson [31] mentioned that "the effect of one parameter may in reality be cancelled out by that of another parameter correlated to the first having the opposite effect." While her paper's subject was related to determining driving patterns based on data, the same principles for the data analysis techniques can be used for fuel consumption data. She narrowed down the number of input variables by combining two variables into one variable, referred to as a factor. Then, each variable and factor were given loadings between 0 and 1 to determine their connection to each other. She used PCA to analyze the data from the vehicles and determine which input variables were the most influential on fuel consumption. She found that heavy acceleration was the main cause of higher emissions and a $30 \%$ to $40 \%$ increase in fuel consumption compared to steady operation. 


\section{Experimental Setup}

\subsection{Experimental Methods}

The design of experiments followed SAE J1321. However, it didn't follow the rules to the letter because the mountainous terrain around West Virginia University (WVU) limited the types of routes in terms of speed and road grade.

The experiment utilized three vehicles (two test vehicles and one control vehicle) of identical make and model, over a period of three months. All vehicles operated with Fuel A (called the baseline fuel in the narrative) for the first and third months of testing. Then, two test vehicles operated with Fuel B (i.e., the test fuel) during the second month, while the control vehicle continued operating with Fuel A. The vehicles were driven on the same routes throughout the three months of testing, but the order of routes changed daily. Moreover, each vehicle accumulated at least 250 miles per day, which depended on the routes, traffic, and weather. The vehicle operators were asked to maintain at least $1 / 4$ of a mile $(1,320$ feet $)$ apart at all times to minimize aerodynamic interference but stayed in radio range so they could exchange information between them at all times. Each vehicle was paired with its own individual trailer for the duration of testing. The trailers had identical aerodynamic contours and were loaded with cement blocks to increase vehicle load during testing.

Fuel flow meters were installed on all three vehicles to measure the actual fuel consumption. The flow meter accuracy of $\pm 0.1 \%$ of the measurement value was well within the desired accuracy of $\pm 0.5 \%$ required by SAE J1321. Additionally, a torque measurement device was installed on each driveshaft to measure the output torque of the vehicle. Moreover, each vehicle had a GPS device to record the latitude and longitude of the vehicle at any moment. The same device also 
recorded the barometric pressure, ambient temperature, relative humidity, and vehicle speed. The following sections details the vehicles and the equipment used for on-road measurements.

\subsection{Vehicle Details}

\subsubsection{Vehicle Specifications}

The vehicles were Class 8 heavy-duty trucks (Freightliner, Model Cascadia day-cab). This particular truck model was chosen due to its popularity with major fleets. A summary of the specifications of each truck is shown in Table 3.1:. Ideally, all vehicles would be the same make, model, and model year. However, Truck 1 was procured due to availability of vehicles at the beginning of the experiment. Truck 1 was selected as the control vehicle so that the test vehicles were identical.

Table 3.1: Vehicle Specifications

\begin{tabular}{|c|c|c|c|c|c|c|c|}
\hline Truck \# & $\begin{array}{l}\text { Test or } \\
\text { Control }\end{array}$ & $\begin{array}{l}\text { Model } \\
\text { Year }\end{array}$ & Make/Model & Engine & $\begin{array}{c}\text { Rated } \\
\text { Power } \\
\text { [HP] }\end{array}$ & Transmission & $\begin{array}{c}\text { Start } \\
\text { Mileage }\end{array}$ \\
\hline 1 & Control & 2018 & \multirow{3}{*}{$\begin{array}{c}\text { Freightliner } \\
\text { Cascadia }\end{array}$} & \multirow{3}{*}{$\begin{array}{l}\text { Detroit } \\
\text { DD13 }\end{array}$} & 525 & \multirow{3}{*}{$\begin{array}{c}\text { Detroit DT12 } \\
\text { Automated } \\
\text { Manual }\end{array}$} & 68,241 \\
\hline 2 & Test & 2016 & & & 500 & & 219,206 \\
\hline 3 & Test & 2016 & & & 500 & & 289,401 \\
\hline
\end{tabular}

\subsubsection{Vehicle Preparations}

A lower variability between datasets is required for experiments that are not expected to produce large differences in the results such as the experiment described here. However, the variability is dependent on the number of variables used to sort the data. For example, the diesel particulate filter (DPF), which is used to remove particulate matter (PM) emissions, affects the 
exhaust flow if not cleaned regularly. The engine manufacturer recommended that the DPF is cleaned when the odometer reaches 300,000 miles. However, the fuel consumption between the vehicles could be skewed if the DPFs were not all cleaned at the same time because the exhaust flows can be different. Therefore, all DPFs were removed and professionally cleaned before the beginning of the experiment. Moreover, to reduce variability in engine operation between trucks, all vehicles were serviced prior to the start of testing including the replacement of all fluids and filters along with fresh grease added to the bearings and U-joints.

\subsubsection{Trailers}

Each vehicle was connected to a trailer for the whole duration of testing. The dry-van trailers (different make and model) were 53-feet long and had identical external contours, so it was assumed that the differences in the aerodynamic forces experienced by each trailer were minimal. There were no trailer skirts. Each trailer was loaded with several large concrete blocks to increase vehicle load similar to the load experienced by a real transporting-cargo fleet. Table 3.2 and Table 3.3 show a summary of each vehicle and trailer's axle and gross weights before and after loading, respectively. As no lower-sized ( $<1,000$ pounds) concrete blocks were available to close the weight gap between the vehicles, the final weights of the vehicles were within $2 \%$ of each other, which was $33 \%$ higher than the $1.5 \%$ requirement from SAE J1321. 
Table 3.2: Vehicle Weights with Empty Trailer

\begin{tabular}{|l|l|l|l|l|}
\hline \multirow{2}{*}{ Truck } & \multicolumn{1}{|c|}{$\begin{array}{c}\text { Steer Axle } \\
\text { Weight [lb] }\end{array}$} & \multicolumn{1}{|c|}{$\begin{array}{c}\text { Drive Axle } \\
\text { Weight [lb] }\end{array}$} & $\begin{array}{c}\text { Trailer Axle } \\
\text { Weight [lb] }\end{array}$ & Gross Weight \\
& 9440 & 11500 & 9980 & 30920 \\
\hline 1 & 10140 & 10760 & 10240 & 31140 \\
\hline 3 & 9340 & 14020 & 12440 & 35800 \\
\hline
\end{tabular}

Table 3.3: Vehicle Weights with Loaded Trailer

\begin{tabular}{|l|l|l|l|l|}
\hline \multicolumn{1}{|c|}{ Truck } & \multicolumn{1}{|c|}{$\begin{array}{c}\text { Steer Axle } \\
\text { Weight [lb] }\end{array}$} & \multicolumn{1}{|c|}{$\begin{array}{c}\text { Drive Axle } \\
\text { Weight [lb] }\end{array}$} & $\begin{array}{c}\text { Trailer Axle } \\
\text { Weight [lb] }\end{array}$ & $\begin{array}{c}\text { Gross Weight } \\
{[\mathbf{l b}]}\end{array}$ \\
\hline 1 & 9400 & 25660 & 21260 & 56320 \\
\hline 2 & 9320 & 24840 & 21040 & 55200 \\
\hline 3 & 9300 & 24120 & 22480 & 55900 \\
\hline
\end{tabular}

\subsection{Routes}

The vehicles were driven on 4 different routes, which are summarized in Table 3.4. Figure 3.1 shows all of the routes geographically, where the color of the route number in Table 3.4 indicates the color of the route outline in Figure 3.1.

Table 3.4: Route Summary

\begin{tabular}{|l|l|l|}
\hline Route \# & Round-Trip Distance [miles] & Average Speed [mph] \\
\hline $\mathbf{1}$ & 23 & 30 \\
\hline $\mathbf{2}$ & 226 & 53 \\
\hline 3 & 158 & 53 \\
\hline $\mathbf{4}$ & 62 & 51 \\
\hline
\end{tabular}


Route 1 goes through downtown Morgantown, WV and continues on the nearby Interstate79 to gather urban driving data in a mix of city roads and highway. Route 2, Morgantown, WV to the Exit 40 along Interstate-79, is mostly highway and included many but consistent grade changes, which, according to Boriboonsomsin et al. [15], was assumed to produce fuelconsumption data similar to a flat route. Route 3, Morgantown to WV Route 50 westbound, is a county highway used to gather highway data at lower vehicle speeds. Finally, Route 4 is a section of Interstate-68 East with a consistent, very-steep grade, where vehicles can reach close to their maximum load.

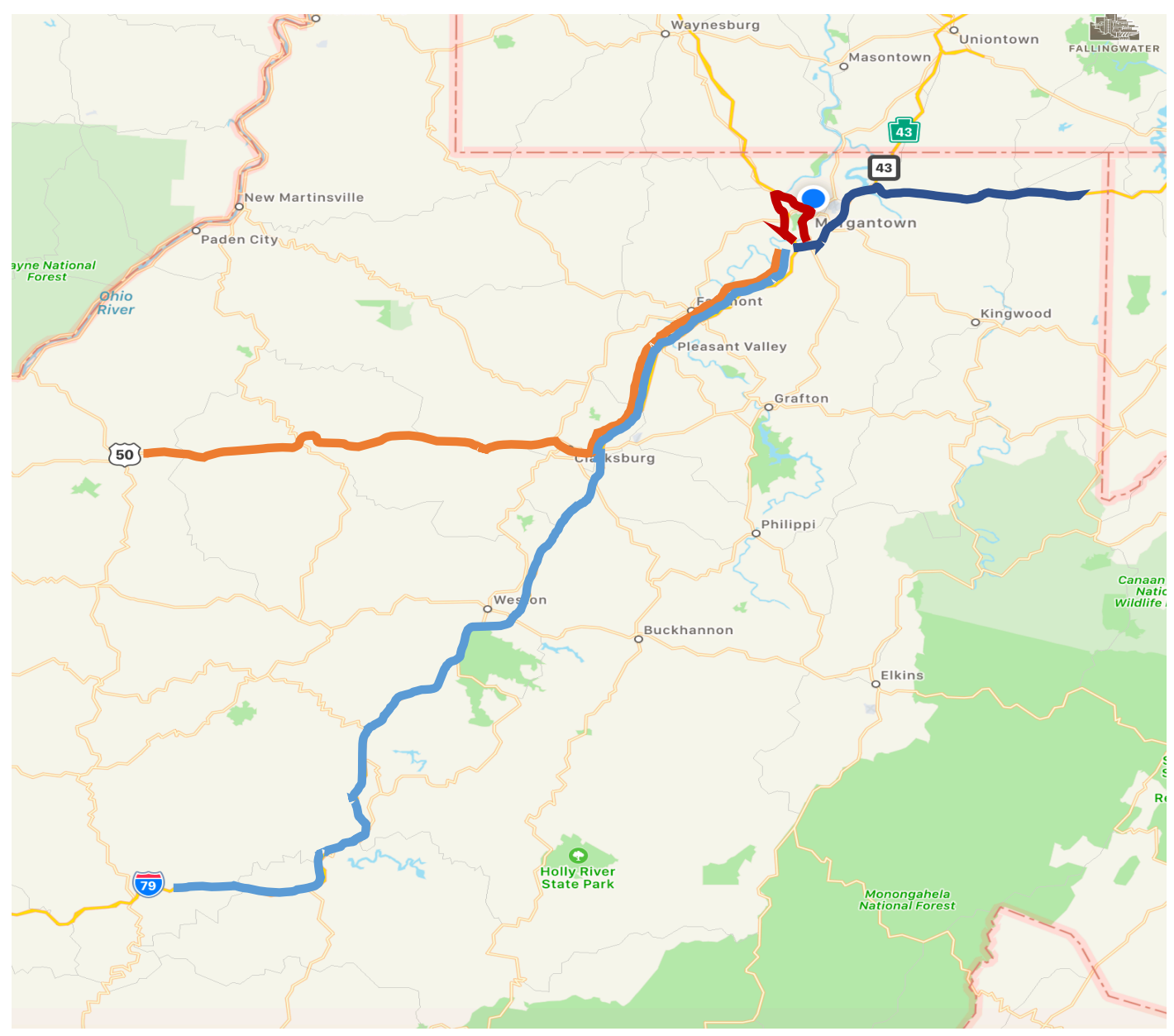

Figure 3.1: Geographical Outline of Routes 
Each day of testing started and ended at WVU CAFEE's Vehicle and Engine Testing Laboratory (VETL). The trucks operated approximately 6 hours per day and the routes were randomly ordered each day, so the results were not biased for variables such as ambient temperature and traffic. The vehicle operators were provided with instructions on how to navigate from one route to another for any combination of routes, so that the transition between routes was consistent.

Recorded on-road the data was binned at the end of each day based on latitude and longitude. Table 3.5 shows the final percentage of each type of route from the total number of data points recorded.

Table 3.5: Percentage of Data Points for Each Route Type

\begin{tabular}{|c|c|}
\hline Route Type & Percentage of Total Data Points [\%] \\
\hline Urban & 5 \\
\hline Local Highway & 6 \\
\hline Interstate & 38 \\
\hline Interstate with Steep Road Grade & \\
\hline
\end{tabular}

\subsection{Vehicle Operator Daily Tasks}

Each day the vehicle operators completed 4 checklists. Two of the checklists were completed at the beginning of the day. The first checklist ensured that data acquisition equipment and vehicles operated properly. The second checklist documented the tire pressure for both the tractor and the trailer. Tire pressure was adjusted if different from the recommended tire pressure (115 psi) to ensure it will not influence the fuel consumption [11]. 
The third checklist was used to document the routes driven each day, in chronological order. Finally, the last checklist documented the weather each day (e.g., if it had rained or snowed at any point during the day; if the winds were light, average, or heavy) and any issues with the vehicle that would need resolved before the next test.

\subsection{Fuel Flow Meters}

\subsubsection{Flow Meter Specifications}

Two positive-displacement flow meters (KRAL, Model OME13) were installed on each vehicle to measure the engine's fuel consumption. One flow meter was installed in the fuel supply line between the engine and the supply tee, which distributes fuel to the engine from each of the two fuel storage tanks housed on the truck. Similarly, the other flow meter was installed in the fuel return line between the engine and the return tee, which distributes unused fuel from the engine back into the tanks. The flow meters were housed in a plastic enclosure to protect them from vibration and the environment. The enclosure was secured to the deck of the truck using $1 / 4$-inch bolts, lock-washers, and nuts. Flow-meter specifications are shown in Table 3.6.

The following describes the fuel flow measurement. Two screws inside the flow meter housing rotated as the fuel passed through them. The volumetric fuel flow rate is determined by counting the frequency at which the screws rotated. The flow meters also measured the fuel's temperature to compensate for temperature effect on fuel density. The flow meter readings were delivered to a separate display (KRAL, Model BEM 500), which performed all the flow calculations and output the corresponding volumetric flow rate at $15^{\circ} \mathrm{C}$. 
Table 3.6: KRAL OME 13 Specifications

\begin{tabular}{|l|l|l|}
\hline \multicolumn{2}{|l|}{ Diameter [inch] } & $1 / 2$ \\
\hline \multirow{2}{*}{ Flow Rate [L/hr] } & $\mathbf{Q}_{\max }$ & 900 \\
\cline { 2 - 3 } & $\mathbf{Q}_{\text {nominal }}$ & 600 \\
\cline { 2 - 3 } & $\mathbf{Q}_{\min }$ & 6 \\
\hline Maximum Pressure [bar] & 40 \\
\hline Temperature Range [ $\left.{ }^{\circ} \mathbf{C}\right]$ & -20 to +125 \\
\hline Viscosity Range [mm $\left.{ }^{2} / \mathbf{s e c}\right]$ & 1 to $1 \mathrm{E} 6$ \\
\hline Accuracy & $\pm 0.1 \%$ \\
\hline K-factor K[P/l] & 1214.0 \\
\hline Frequency at Q & 202 \\
\hline
\end{tabular}

\subsubsection{Flow Meter Testing}

The flow meters were installed on the supply and return lines of an engine (Cummins, Model ISX-15) attached to a dynamometer prior to being installed on the vehicles. A Supplemental Emissions Test (SET) engine cycle was completed because it has 13 "modes" which allow the engine to operate at steady-state for different combinations of engine speed and load. The SET engine cycle was selected because steady-state operation is the condition where the least variability can occur. Therefore, the differences between fuel measurement devices will be maximum during steady-state operation. Table 3.7 shows a summary of the engine speed and load combinations for each mode of the SET engine cycle. Each mode consists of 150 seconds of engine operation. 
Table 3.7: Summary of SET Cycle Modes

\begin{tabular}{|c|c|c|}
\hline Mode & RPM & $\begin{array}{c}\text { Torque } \\
{[\%]}\end{array}$ \\
\hline 1 & 600 & 8 \\
\hline 2 & 1290 & 55 \\
\hline 3 & 1550 & 41 \\
\hline 4 & 1550 & 55 \\
\hline 5 & 1290 & 41 \\
\hline 6 & 1290 & 55 \\
\hline 7 & 1290 & 25 \\
\hline 8 & 1550 & 55 \\
\hline 9 & 1550 & 25 \\
\hline 10 & 1800 & 55 \\
\hline 11 & 1800 & 25 \\
\hline 12 & 1800 & 55 \\
\hline 13 & 1800 & 41 \\
\hline
\end{tabular}

The fuel consumption values from the ECU and flow meters were integrated for each mode and compared by calculating the percent difference between the two values using Equation (6):

$$
\% \text { Diff }=\frac{F C_{K R A L}-F C_{E C U}}{F C_{E C U}} * 100
$$

where $F C_{K R A L}$ and $F C_{E C U}$ are the total fuel consumption values according to the flow meters and the ECU, respectively. Figure 3.2 shows the percent difference values for each mode of the SET engine cycle. These percent error values are the minimum error that may occur in on-road testing for each steady-state condition because all variables were held constant. Modes 1 and 7 have the largest percent differences, which is likely due to the low power and engine speeds used for the modes, which imposes fuel consumption rates near the lower bound of the flow meter measuring range. 


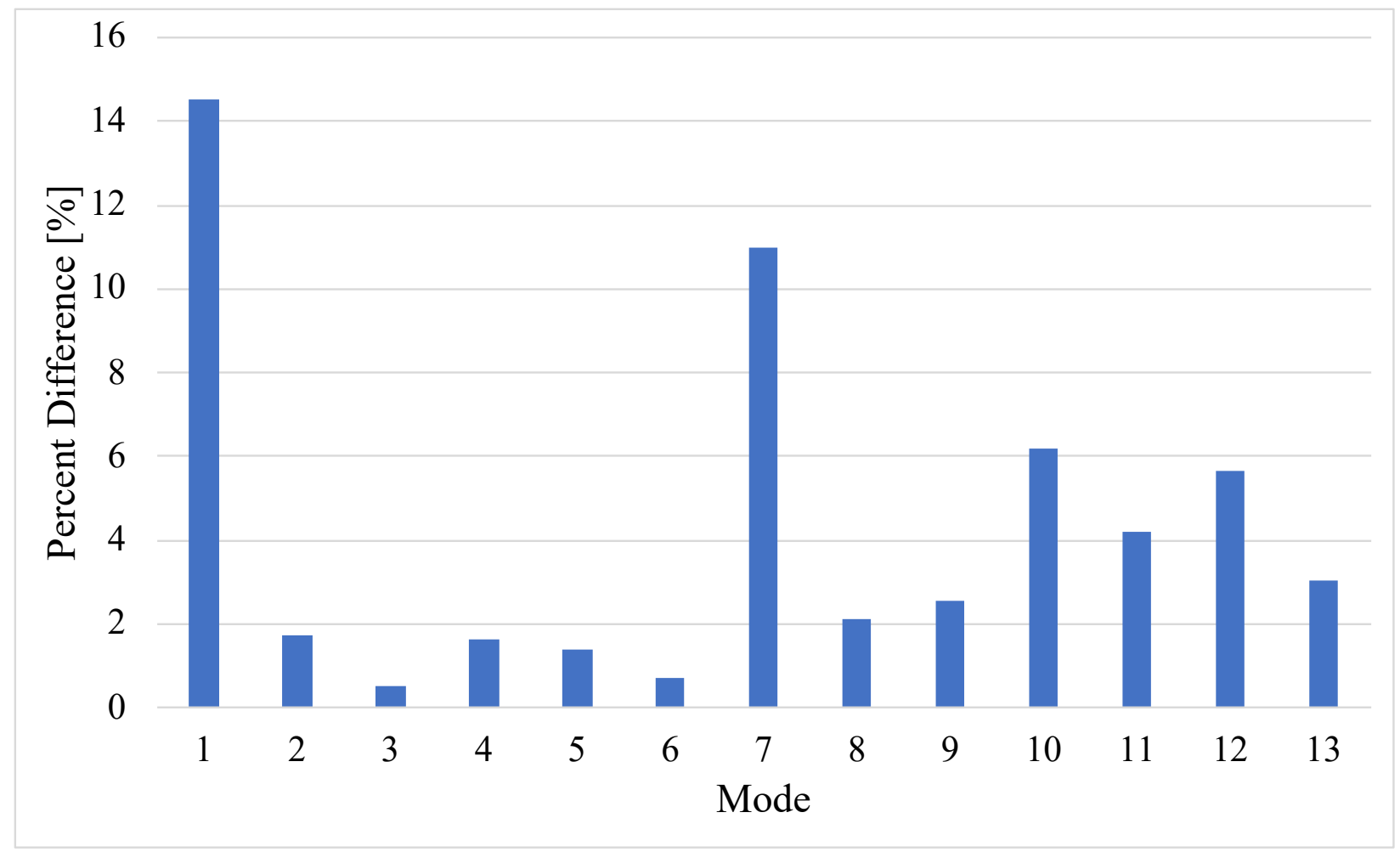

Figure 3.2: Percent Difference of ECU and KRAL Fuel Consumption for Each Mode of SET Cycle

An FTP engine cycle was also completed to determine differences between the fuel consumption according to the flow meters and the ECU for transient engine operation. Figure 3.3 shows the instantaneous fuel consumption rates according to the KRAL flow meters and the ECU have consistent positive correlations with engine power. However, the KRAL rates have more variation than the ECU, especially in the low power regions $(0-150 \mathrm{HP})$. Additionally, there is separation between the ECU and KRAL values at the very top of the power range (500 - $600 \mathrm{HP})$. 


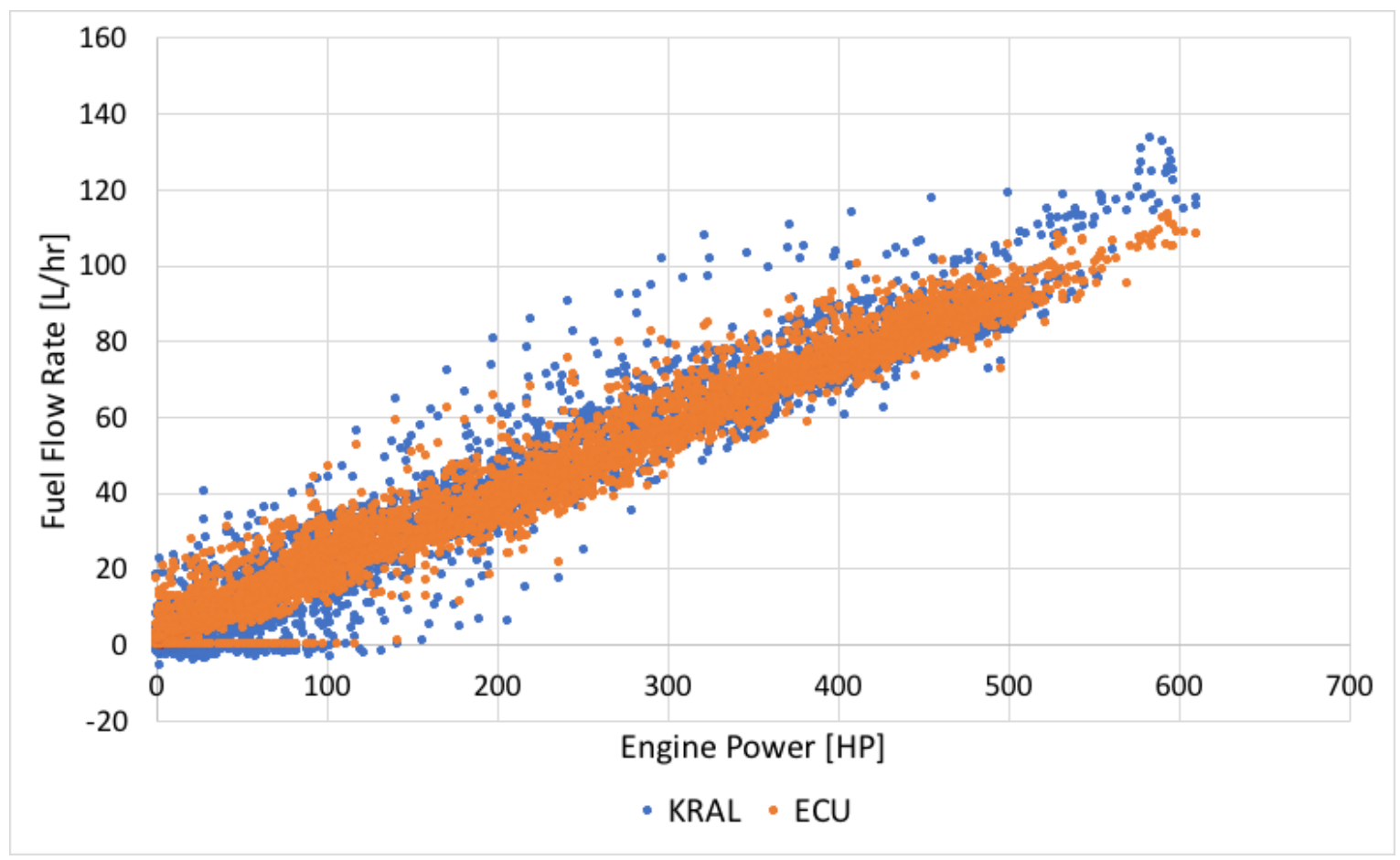

Figure 3.3: Comparison of ECU and KRAL Flow Rate for Varying Engine Power

Because Figure 3.2 and Figure 3.3 show differences between the flow meters and the ECU in certain regions, the fuel consumption data from the FTP cycle was integrated to determine the overall fuel consumption for the entire engine cycle. The integration was completed using Equation (7):

$$
F C_{\text {total }}=\frac{1}{36000} * \sum_{\mathrm{i}=1}^{\mathrm{n}} F C_{\text {rate }, i}
$$

where $n$ is the total number of data points and $F C_{\text {rate }, i}$ is the instantaneous fuel consumption flow rate for data point $i$. The integration was completed for both the ECU and flow meter flow. rates. Then, these values were compared by calculating the percent difference between them using Equation (6). Table 3.8 shows the total fuel consumption values calculated according to the KRAL flow meters and the ECU along with the percent difference between them. 


\section{Table 3.8: Percent Difference Between Fuel Consumption According to KRAL and ECU}

\begin{tabular}{|c|c|c|}
\hline $\begin{array}{c}\text { FC from KRAL } \\
\text { Flow Meters } \\
{[\mathbf{L}]}\end{array}$ & $\begin{array}{c}\text { FC from ECU } \\
{[\mathbf{L}]}\end{array}$ & $\begin{array}{c}\text { Percent } \\
\text { Difference [\%] }\end{array}$ \\
\hline 6.0212 & 6.0236 & 0.03887 \\
\hline
\end{tabular}

\subsubsection{Fuel Hoses}

The supply and return fuel lines on the truck were high temperature fuel line tubing (Parker, Model HTFL) with compression fittings at all connections. The supply fuel hose had a 5/8-inch outer diameter (OD) and the return fuel hose had a 1/2-inch OD. To extend the fuel line to the flow meters, 5/8-inch and 1/2-inch inner diameters (ID) rubber hose (Eaton) was attached to the supply and return fuel lines, respectively. Larger IDs were chosen so the fuel flow was not restricted. Additionally, rubber hose for barbed fittings was chosen over the high temperature fuel line tubing due to availability of both hose and compression fittings. Therefore, each flow meter was fitted with hose barbs with the appropriate dimensions for the respective supply and return hoses.

\subsubsection{Flow Meter Time Alignment}

Because the flow meters were located away from the fuel injectors, on the deck of the truck, the instantaneous fuel consumption values collected by the flow meters were not time aligned with the ECU data. As a result, instantaneous fuel consumption values from the flow meters were not comparable to the ECU's fuel consumption values during transient (urban) vehicle operation because of the frequent changes in fuel consumption. However, during steady (highway) vehicle operation, the instantaneous ECU fuel consumption and the flow meters' instantaneous net flow calculations had similar variations but were slightly misaligned. Therefore, a time alignment 
algorithm was used on the acquired data, which delayed the supply flow meter data and advanced the return flow meter data to determine the actual net fuel consumption values. The phenomena is very complex as the actual advance and delay were dependent on engine operation (i.e., they were not constant)

The algorithm was based on the conservation of mass principle, as shown in Eq (8):

$$
\dot{\forall}=\mathrm{A}_{\mathrm{c}} \mathrm{V}
$$

where $\dot{\forall}$ is the volumetric flow rate, $A_{c}$ is the cross-sectional flow area, and $V$ is the velocity of the fluid. Therefore, the length of time a fluid particle takes to travel a certain distance through a tube can be calculated using Equation (9), where $L$ is the length of the tube.

$$
\text { Travel Time }=\frac{\mathrm{A}_{\mathrm{c}} \mathrm{L}}{\dot{\forall}}
$$

In this case, the lengths and inner diameters of the supply and return hoses were used, along with the volumetric supply and return flow rates found by the flow meters at each 0.1 -second time interval. However, this method could not accurately time-align the data, so the total fuel consumption over a given time interval was used rather than instantaneous values. Specifically, the volumetric flow rates of the supply and return flow meters were integrated over a time interval. The total fuel consumption for a certain time interval was the difference between the integrated supply value and integrated return value.

\subsection{Torque Meter}

\subsubsection{Torque Meter Specifications}

Engine torque transmitted was measured with a torque sensor (Accumetrics, Model AT4500) installed on the driveshaft. The sensor includes several components. The first component is 
a transmitter, which is mounted to the driveshaft using a specially designed strap with counterweights. The transmitter was connected to a 350-ohm full-bridge strain gage, which was mounted to the surface of the driveshaft using a very complex procedure (see torque-meter manufacturer website for the installation procedure). The connection between the strain gage and the torque transmitter followed the recommendation for measuring torque on a shaft [32], which also suggested the proper strain gage orientation of the shaft based on the direction of shaft rotation.

The transmitter was powered via induction by a brass ring. The brass ring was mounted such that it was centered around the driveshaft and allowed for the transmitter to rotate with the driveshaft freely, without colliding with the metal surrounding it. The brass ring also received the signal from the transmitter and sent it to a tuning/amplifying box. However, as the signal can be influenced by the interference with the surrounding metal, this work followed the procedure for tuning the signal to the optimal level of amplification mentioned in the torque meter manual [33]. The tuning procedure was completed after the torque meter was installed, but before the experiment began. The tuning box is connected to the receiver via $1 \mathrm{BNC}$ and $1 \mathrm{TNC}$ cables, so that the connections on the back of the receiver are not confused. The BNC cable receives the signal from the brass ring and the TNC cable provides the power to the ring from the receiver, which then is sent to the transmitter mounted on the driveshaft.

\subsubsection{Torque Meter Output}

A 16-pin serial connector gathered the output of the receiver, but only 6 pins were used. Two of the pins facilitated a current output which was used for tuning purposes, another two pins provide a voltage output, and the last two pins provide the output signal as a frequency. The frequency output ranged from $5 \mathrm{kHz}$ to $15 \mathrm{kHz}$, so $10 \mathrm{kHz}$ indicates zero torque on the shaft and 
$5 \mathrm{kHz}$ and $15 \mathrm{~Hz}$ indicate the maximum torque in the negative and positive directions, respectively. The frequency output was the only output utilized in this work. Therefore, the output of the torque meter was not in engineering units of torque (i.e., pound-foot or Newton-meter).

\subsubsection{Torque Meter Calibration}

The torque meter must be calibrated before testing to convert the frequency output to engineering units. The procedure recommended by the torque meter manufacturer involved the removal of the driveshaft from the vehicle at the U-joints and creating a station where the rotation can be locked on one end of the driveshaft, while the other end can be free to rotate. A moment arm should be mounted to the end that rotates freely and calibrated weights should be added to produce a known torque on the driveshaft. The frequency given by the torque meter for each calibration torque should be recorded and a linear regression would determine the actual torque value for each frequency value given by the torque meter.

However, removing the shaft was not possible during this study. The torque signal was calibrated with the truck parked inside a garage on a level floor. The parking brake was disabled so that the rear wheels were free to move and a prybar was used to prevent the driveshaft from rotating at the transmission end. Ideally, the transmission end of the driveshaft would be locked mechanically, not with a prybar, because the prybar allows the potential for some rotation or flexing of the driveshaft. Blocks were placed in front of the front wheels to prevent the truck from moving. Then, the front rear axle was elevated, such that the wheels were just off the ground. A long piece of rectangular bar stock was wedged in the yoke of the drive shaft. The approximate horizontal distance from the center of the driveshaft to the end of the bar stock was approximately 31.25 inches. Then, calibration weights were applied incrementally to the end of the bar stock and the weight was multiplied by the horizontal distance to determine the torque applied on the shaft. 
The output frequency was found at each increment by using a multimeter in the frequency mode where the positive and negative leads were placed on the positive and negative frequency output wires, respectively. The torque and frequency were recorded at each increment and a linear regression determined the best fit. This line of best fit was used to calculate the measured driveshaft torque in both Newton-meters and pound-feet. However, as the transmission end of the driveshaft could not be locked mechanically, the exact error in engineering units between the actual and measured torque value is unknown.

\subsubsection{Strain Gage}

\subsubsection{Strain Gage Purpose}

The strain gage measured the driveshaft torque. The transmitter receives the signal from the strain gage via a 4-pin connector. As torque is applied to the driveshaft, the strain gage flexes and changes its resistance, thus changing the signal sent to the transmitter. The torque meter manufacturer recommended the 350-ohm strain gage (Micro-Measurements, Model CEA-03250US-350).

\subsubsection{Strain Gage Installation}

A location for the strain gage had to be determined before the transmitter and strain gage could be mounted to the shaft. Since the brass ring must remain centered around the transmitter at all times without touching, movement of the driveshaft in that particular region must be minimal. Unfortunately, the suspension caused significant lateral movement of the driveshaft near the end 
of the driveshaft at the rear axle. Therefore, the strain gage and transmitter were mounted at the end of the driveshaft closest to the transmission, where the lateral movement was minimum.

The strain gage manufacturer procedure for mounting the strain gage to the shaft was followed [34]. First, the surface needed to be prepared for the adhesive, so the paint was removed from the area of the driveshaft where the strain gage was to be applied using a handheld electric grinder. This 3-inch wide section around the entire circumference of the driveshaft was then cleaned with brake parts cleaner. Next, 320 and 400-grit silicon-carbide sandpaper were used in succession in combination with a conditioner provided by the strain gage manufacturer to rid the area of any unevenness. Then, the desired location of the strain gage was marked. Finally, the area was scrubbed using the conditioner, then flushed with a neutralizer (provided by the strain gage manufacturer) to balance the $\mathrm{pH}$ of the surface and conclude the surface preparation process.

After the surface was prepared, the strain gage was also prepared for application. The flat surface where the strain gage could be placed was cleaned and then flushed with the neutralizer. For convenience, the top surface of the enclosure for the flow meters was used. The strain gage was removed from its packaging using forceps, which were used to grip the strain gage at one of the corners so that the strain gage was not damaged. Gripping the strain gage at any other location makes the strain gage unusable because any contact with the Wheatstone bridge can cause irreversible damage. The strain gage was placed on the flat surface and a piece of packing tape was placed and smoothed on the top side of the strain gage to be able to transfer the strain gage to the driveshaft without damage. However, before the packing tape was applied, the tape was placed and removed from a clean surface in order to remove a portion of the adhesive, so that the tape would more easily separate from the strain gage after the adhesive finished curing. Once the strain gage was moved to the shaft, it was aligned based on the markings completed during the surface 
preparation process and was taped in place. Then, the tape was peeled back to expose the back side of the strain gage.

Following the same strain gage installation procedure, catalyst (M-Bond 200) was applied to the back of the strain gage and allowed to dry. Finally, a small amount of catalyst adhesive was applied at the base of the strain gage and the tape was folded back to its original position, such that the bottom of the strain gage was against the shaft. The glue was distributed using the author's thumb, and pressure was applied to the strain gage for 1 minute to ensure strong adhesion. However, the high centrifugal forces experienced by the strain gage during vehicle operation caused the strain gage to peel away from the shaft, which meant that the torque meter signal was not always reliable.

To remedy the issue, the strain gage manufacturer was contacted to determine if there was an alternative adhesive that would allow the strain gage to remain adhered to the driveshaft for the entirety of testing. Based on their advice, the fiberglass-cloth installation procedure for the strain gages was followed [35]. The procedure suggests using a special adhesive (M-Bond GA-61), which has to cure at a temperature of $350^{\circ} \mathrm{F}$. Because the driveshafts could not be easily removed from the trucks and placed in an oven to cure, a substitute adhesive (M-Bond AE-10) was used instead. This adhesive can cure at a temperature as low as $70^{\circ} \mathrm{F}$ for a duration of 6 hours, which was feasible by positioning all of the trucks inside the garage when the adhesive was curing. The strain gage manufacturer suggested using a heat gun to increase the surface temperature of the shaft to expedite the curing process, but the heat guns readily available produced too much heat and could not be adjusted, so heat lamps were procured to heat the adhesive. The curing time for different temperatures was explained in the adhesive packaging.

The procedure for the strain gage surface preparation remained the same as previously practiced. Then, the new adhesive (M-Bond AE-10) was applied to back of the strain gage and the 
strain gage was pressed against the shaft. Pressure was applied to the strain gage for the duration of the curing process using a 4-inch wide piece of rubber that was wrapped around the driveshaft followed by a hose clamp to hold it in place and apply pressure. Then, a heat lamp was situated above the driveshaft, directly above the strain gage. The heat lamp was switched on and off periodically because the temperature could reach over $400^{\circ} \mathrm{F}$ if it was left on continuously, which would melt the rubber. The temperature of the rubber was monitored using a non-contact infrared thermometer. The heat lamp was switched on when the temperature dropped below $100^{\circ} \mathrm{C}$ and was switched off when the temperature was above $200^{\circ} \mathrm{C}$.

\subsubsection{Strain Gage Temperature Compensation}

As the driveshaft material (presumably steel) and the strain gage material (molybdenum) were not the same, the voltage output may differ with changes in ambient temperature because of the different thermal expansion coefficients [36]. The strain gage manufacture provides a technical note that details the procedure to compensate for this difference [36]. A Matlab program was developed using the technical note to calculate the changes in voltage because of temperature material differences. Table 3.9 shows the specifications for the molybdenum strain gages.

Table 3.9: Strain Gage Specifications

\begin{tabular}{|l|l|}
\hline Model Number & CEA-03-250US-350 \\
\hline Grid Resistance $[\mathbf{O h m s}]$ & $350.0 \pm 0.4 \%$ \\
\hline Thermal Coefficient of Gage Factor $\left[\mathbf{\%} / \mathbf{1 0 0}{ }^{\circ} \mathbf{C}\right]$ & $+1.2 \pm 0.2$ \\
\hline Gage Factor $\mathbf{a} \mathbf{2 4}^{\circ} \mathbf{C}$ & $2.09 \pm 1.0 \%$ \\
\hline Transverse Sensitivity & $(+0.8 \pm 0.2) \%$ \\
\hline
\end{tabular}


The thermal output of a strain gage refers to the change in signal due to changes in temperature [36]. Equation (10) (given by the strain gage manufacturer) determines the thermal output of the strain gage based on the temperature [36] and the coefficients specified in Table 3.10.

$$
\varepsilon_{\mathrm{T} / \mathrm{O}}=\mathrm{A}_{0}+\mathrm{A}_{1} \mathrm{~T}+\mathrm{A}_{2} \mathrm{~T}^{2}+\mathrm{A}_{3} \mathrm{~T}^{3}+\mathrm{A}_{4} \mathrm{~T}^{4}
$$

\section{Table 3.10: Thermal Output Coefficients}

\begin{tabular}{|l|l|l|}
\hline Coefficient & $\begin{array}{l}\text { Thermal Output Coefficient for } \\
\text { Temperature in Fahrenheit }\end{array}$ & $\begin{array}{l}\text { Thermal Output Coefficient } \\
\text { for Temperature in Celsius }\end{array}$ \\
\hline$A_{0}$ & $-2.67 \mathrm{E}+2$ & $-1.0 \mathrm{E}+2$ \\
\hline$A_{1}$ & $+6.14 \mathrm{E} 0$ & $+6.81 \mathrm{E} 0$ \\
\hline$A_{2}$ & $-4.14 \mathrm{E}-2$ & $-1.05 \mathrm{E}-1$ \\
\hline$A_{3}$ & $+1.00 \mathrm{E}-2$ & $+5.03 \mathrm{E}-4$ \\
\hline$A_{4}$ & $-1.17 \mathrm{E}-7$ & $-1.11 \mathrm{E}-6$ \\
\hline
\end{tabular}

For example, when the temperature is given in degrees Celsius, the thermal output can be calculated using Equation (11):

$$
\varepsilon_{\mathrm{T} / \mathrm{O}}=-100.0+26.81 \mathrm{~T}-0.105 \mathrm{~T}^{2}+0.000503 \mathrm{~T}^{3}-0.00000111 \mathrm{~T}^{4}
$$

Equation (12) was used to determine the thermal output of the strain gage for a change in temperature and when the material of the gage and substrate are not the same [36]. For Equation (12), $\varepsilon_{\mathrm{T} / \mathrm{O} \text {,shaft }}$ is the thermal output compensated for differences in temperature and material, $\varepsilon_{\mathrm{T} / \mathrm{O} \text {,gage }}$ is the thermal output based on the gage material found using Equation (10), $\alpha_{\text {shaft }}$ is the thermal expansion coefficient of the shaft material, $\alpha_{\text {gage }}$ is the thermal expansion coefficient of the gage material, and $\Delta \mathrm{T}$ is the change in temperature from the reference temperature, which is the temperature at which the strain gage was applied to the shaft [36]. 


$$
\varepsilon_{\mathrm{T} / \mathrm{O}, \text { shaft }}=\varepsilon_{\mathrm{T} / \mathrm{O}, \text { gage }}+\left(\alpha_{\text {shaft }}-\alpha_{\text {gage }}\right) \Delta \mathrm{T}
$$

Then, using Equation (13) for a full bridge strain gage, the change in voltage was calculated, where $\mathrm{V}_{\mathrm{EX}}$ is the excitation voltage and GF is the gage factor of the strain gage [37].

$$
\Delta \mathrm{V}=-\mathrm{V}_{\mathrm{EX}} \cdot \mathrm{GF} \cdot \varepsilon_{\mathrm{T} / \mathrm{O}, \text { shaft }}
$$

The strain gage manufacturer suggests compensating for changes in gage factor as well as changes in strain for a given temperature difference [36]. However, from Table 3.9, the change in

gage factor is $1.2 \%$ per $100^{\circ} \mathrm{C}$, which is 0.02717 per $100{ }^{\circ} \mathrm{C}$. Since the local driveshaft temperature differences the trucks experienced throughout the testing phase was never near $100^{\circ} \mathrm{C}$, the gage factor was not compensated for the changes in ambient temperature.

From these calculations, for a temperature difference of $25^{\circ} \mathrm{C}$ and assuming a steel shaft, the change in voltage is $\sim 3 \mathrm{mV}$ or $\sim 3 \mathrm{~Hz}$ for the frequency output. Therefore, temperature compensation was not a significant error source since $3 \mathrm{~Hz}$ was only $0.03 \%$ of the entire $5 \mathrm{kHz}$ to $15 \mathrm{kHz}$ frequency output range.

\subsubsection{Torque Meter Testing}

Similar to the flow meters, the torque meter was installed on the output shaft of an engine (Cummins, Model ISX-15) attached to a dynamometer prior to being installed on the vehicles. A SET engine cycle was not used because the torque meter was not integrated into the data acquisition system to collect the data. Instead, the engine was manually controlled to cause steady state operation for different combinations of engine speed and load. Then, the voltage output of the torque meter was measured using a multimeter to ensure the output was a steady signal. A linear relationship between the engine torque and the torque meter signal was observed. 


\subsection{Portable Activity Monitoring System (PAMS)}

The last equipment installed on the trucks was the portable activity monitoring system (PAMS). The PAMS design is proprietary, but its function was to collect GPS data, ambient temperature, relative humidity, altitude (based on GPS), and barometric pressure. The unit also stored all the data from the vehicle's controller area network (CAN) bus, flow meters, and torque meter when the vehicle was keyed on, at a frequency of $10 \mathrm{~Hz}$. The data was stored on a mini SD card and it could be accessed remotely using an access point. The PAMS was mounted to the roof of the cab, underneath the air dam, using two large suction cups that were checked every morning to ensure they were properly secured.

\subsection{Printed Circuit (PC) Board for Data Acquisition}

In order to incorporate the data from the flow meters and the torque meter onto the CAN bus, a printed circuit (PC) board was designed to both power the sensors and transform the data to be compatible with the CAN bus.

Screw terminals were used to connect the flow meters, torque meter, vehicle power, and the CAN bus to the board. Only the CAN's high and low wires were introduced to the board, all other wires in the RJ45 cable remained uninterrupted. Therefore, the board acted as a "tee" in the CAN bus, such that the CAN data from the vehicle enters the board, new data from the sensors are added and the data continues on to the PAMS for addition of its data and for data storage. A Teensy 3.5 evaluation board was used to convert the raw signals from both the flow meters and the torque meter to CAN signals. The Teensy 3.5 was selected because of its ability to capture signals at high frequencies. The torque meter signal, at its highest frequency, can reach 15,000 Hz. The Teensy can capture the entire signal so the user is able to evaluate the minimum, maximum, and average 
frequency found in each 0.1 second window of time, since the rest of the data is captured at $10 \mathrm{~Hz}$. The Teensy was programmed with an Arduino code to complete this task. The signal from the flow meters is an analog signal between $0 \mathrm{~V}$ and $12 \mathrm{~V}$. A logic transceiver was used to convert the signal from the flow meters to a logic-based signal (a signal between $0 \mathrm{~V}$ and $3.3 \mathrm{~V}$ ), which is required to be interpreted by the Teensy.

The 12V supplied by the vehicle was used to power not only the board's components, but also the flow meters and the torque meter. However, the flow meters required 24-Volts for adequate power, so a RECOM 24-Volt power supply was used for amplification to adequately power the flow meters. On the other hand, the components on the PC board required 3.3-Volts of power, so a RECOM 3.3-Volt power regulator was used to decrease the voltage of the input power to deliver to the components. A 4-Amp fuse was included on the board to protect all components. However, this is not the only protection; there were also 5-Amp fuses at the power source and on the enclosure, which houses the PC board, the receiver for the torque meter, and the display for the flow meters.

Figure 3.4 shows the enclosure (Bud Industries, Model TR-6103) used to house all the equipment. The front panel was removable, so all the equipment can be fitted inside, and the front panel concealed all the wires. Additionally, the front panel was machined so that the flow meter display, torque meter receiver, and panel-mount connectors could be mounted to the front panel. The panel-mount connectors were used for all connections that needed to be made internally, so that the enclosure never had to be opened, unless there was an issue with the equipment. These connections included the $\mathrm{BNC}$ and $\mathrm{TNC}$ cables for the torque meter, a 15-pin serial connector to connect the flow meters to their display, two RJ45 ports to carry the CAN signal, and the power input. The power was supplied to the enclosure from the cigarette outlet. The enclosure was placed on the passenger's seat inside the cab of the vehicle and strapped in place to avoid both excessive 
vibration and movement of the enclosure during the acceleration and deceleration of the vehicle, as seen in Figure 3.4.

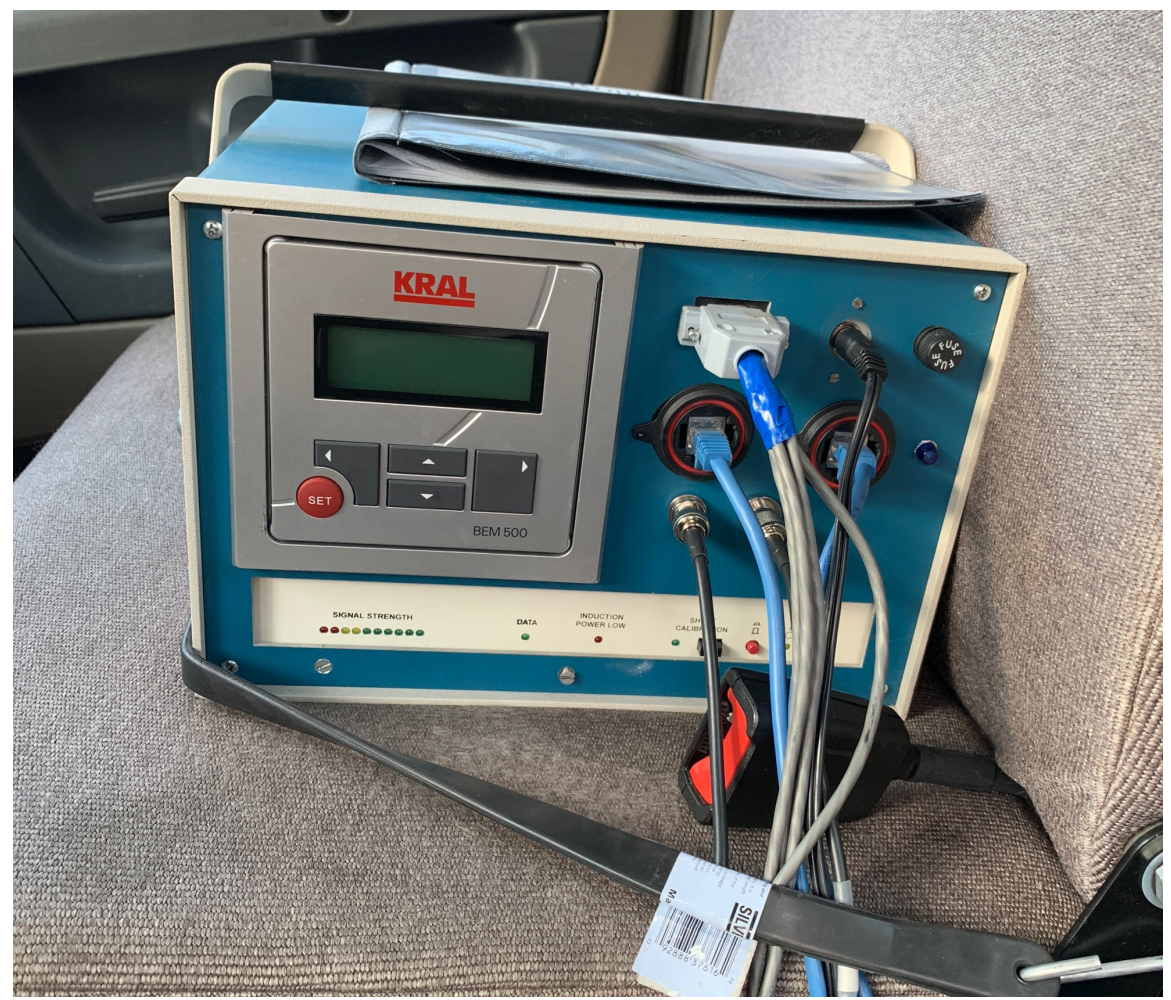

Figure 3.4: Enclosure Placement in Vehicle 


\section{Data Collection and Analysis}

The thesis cannot show actual results due to the proprietary nature of the data. However, it describes the methodology used for the collection and data analysis. The data analysis can be used to determine variable effects in any large dataset regardless of application.

\subsection{Data Collection and Storage}

Each test session created a file that was stored by the PAMS, then accessed remotely later using an access point. The beginning of a test session was defined by the "key on" signal when the vehicle's engine is started, and the end of a test session was defined as 15-minutes after the engine has been "keyed off." If the engine was restarted before the 15-minutes has expired, the session remained active until the 15-minutes of no engine activity was achieved. Each test session collected and wrote data from the sensors and the vehicle's ECU in one file, while the GPS data was stored in a separate file. These files were loaded into a proprietary program and merged together for time-alignment between the ECU and PAMS data. The merged file was loaded into Matlab but not all ECU variables were used for analysis. Only certain variables were selected based on the Literature and CAFEE previous studies. These variables included engine speed, engine torque, vehicle speed, intake temperature, EGR mass flow rate, oil temperature, coolant temperature, fuel rate, transmission gear, accelerator pedal position, exhaust temperature, and all the ambient condition variables collected by the PAMS. Each vector of a desired variable was stored within a structure in Matlab using 'dot-indexing' and the structure was saved as a .mat file for each test session.

To keep the data separated, each vehicle's files were stored in a separate folder and the file name contained the vehicle's identification number, test session number, and date of testing. The 
data was separated into folders based on fuel and weather during the test session (e.g., rain, snow etc.) as mentioned in the drivers reports.

\subsection{Data Reduction}

Certain data points were omitted during the fuel consumption analysis to avoid skewed averages and correlations. This is because the ECU either significantly reduce or completely stop fuel injection when the vehicle is travelling downhill (also known as engine cutoff). Therefore, these data points should not be included in the analysis because the average fuel consumption rate for similar conditions would be skewed toward zero if they were included. Engine cutoff were the data points where the vehicle was moving at a cruising speed but there was zero fuel injection. It can further be confirmed if sensor data (e.g., altitude or barometric pressure) shows that the vehicle is travelling downhill. In the analysis for this work, the engine cutoff points were identified as points where the vehicle speed was greater than 20 kilometers per hour and the ECU fuel rate was equal to zero when rounded to 2 decimal points.

Data points for engine idling were also deleted because the fuel consumption during idling may be affected by engine or cabin accessories. If analysis on both idling and constant engine operation is desired, separate analyses should be conducted instead of including all points in one analysis. Additionally, points with negative torque values were also omitted.

Finally, this study discarded the data acquired in days where it rained or snowed at any point of the day because wet roads influence the friction coefficient on the tires, which would bias the results. Therefore, only data acquired during days with dry roads were considered for analysis.

There were approximately 4.5 million data points collected per month for each vehicle. Fifty-five percent of the total number of points was discarded after the data reduction procedures 
mentioned above. As a result, the analysis was based on approximately 2 million data points per month for each vehicle.

\subsection{Coefficient of Variation (COV)}

For engines tested on a dynamometer, the variation in fuel consumption can be explained mostly by the engine speed and load because most of the other variables are held constant. However, for on-road testing, most variables cannot be held constant, so a significant variation in the data is expected. Therefore, once the data is compiled, the amount of variability should be determined for different conditions to determine where the least and most variation lies in the data. The instantaneous fuel consumption rate should be separated into bins based on the route and then by different combinations of engine speed and power. Then, the coefficient of variation (COV) should be calculated for each bin using Equation (14):

$$
\operatorname{COV}=\frac{\sigma}{\mu} * 100 \%
$$

where $\sigma$ is the standard deviation and $\mu$ is the mean of each bin.

A contour plot can be generated to visually determine where the highest and lowest COV values are. This is important if the experiments need to determine small differences between datasets because the regions with the lowest variation are the most likely regions to suggest a difference with the highest confidence interval. Therefore, the data from sections with the lowest variation should be examined first. This is especially helpful if the entire dataset is too large to be analyzed all at once due to lack of processing power.

Figure 4.1 shows an example contour plot of the COV for the ECU broadcasted instantaneous fuel consumption at different engine speed and power. The color bar on the right side of the figure indicates the COV as a percentage for each bin. In this case, it shows that the 
regions with the lowest variability are in the high-power (>50\%) regions and medium engine speeds (1000 to $1400 \mathrm{RPM}$ ). It also shows that low-power regions have high COVs for any engine speed except for idling (600 RPM).

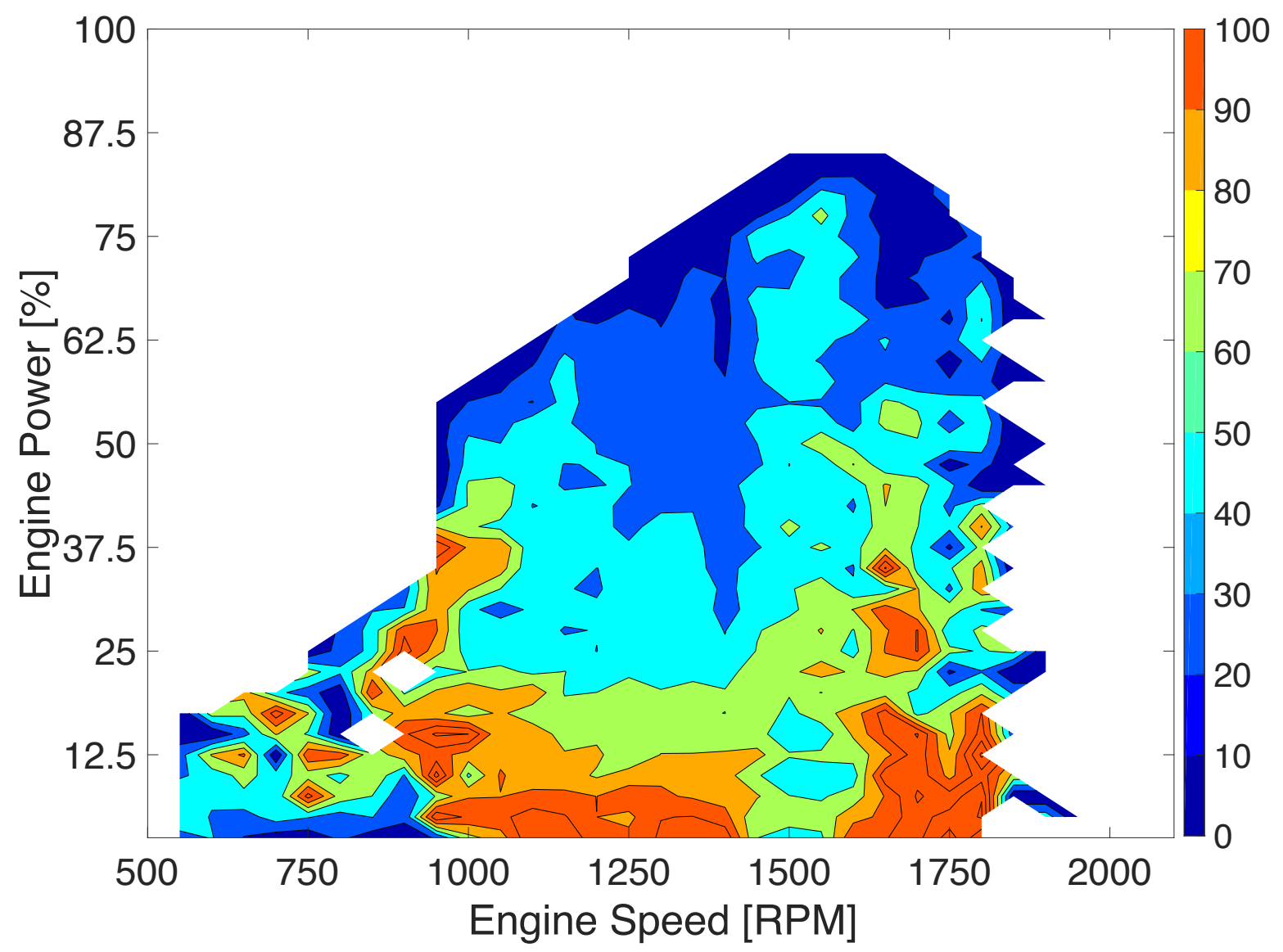

Figure 4.1: Example COV Contour Plot

\subsection{Determining Data Concentration}

In addition to COV, the number of data points for every engine speed and power combination should be found and plotted using a contour plot similar to Figure 4.1. Areas with the largest number of data points should be investigated first in the data analysis process. As data is excluded 
and separated into bins, the areas with the largest number of points are most likely to have a statistically significant number of points after binning. Figure 4.2 is an example contour plot, where the data was separated by engine speed and power and the number of data points was found, which is indicated by the color bar on the right side. It shows that the greatest concentration of points lies for engine power and speed between 300 and 450 HP and 1000 and 1700 RPM, respectively.

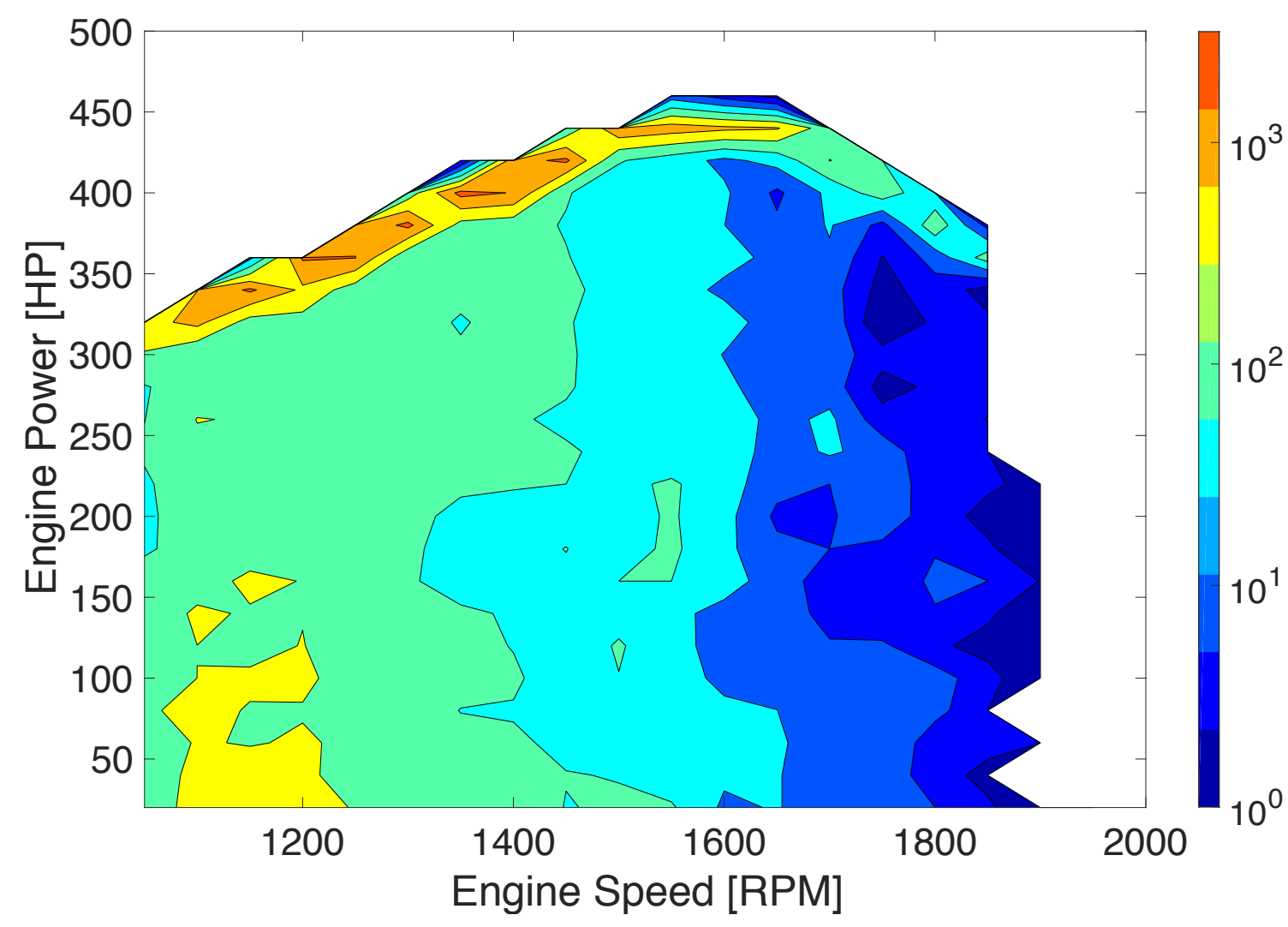

Figure 4.2: Number of Data Points Contour Plot Example 


\subsection{Data Sampling}

When computational capability is reduced, data samples should be analyzed rather than the entire population, if the entire dataset is desired to be analyzed at one time. Sampling techniques as those discussed in Section 2.3.1 can be used for datasets too large to be analyzed at once.

\subsection{Principal Component Analysis}

Principal component analysis (PCA) was applied to each dataset to determine which variables were having the most influence on the fuel consumption data. Therefore, in this work, PCA was performed three times for each vehicle, one PCA for each month of testing. It allowed the user to compare the effect of each variable on the variation for each dataset from month-tomonth. There are several different programs that can perform PCA, including JMP and Matlab. If the number of rows in the dataset is greater than the capability of Excel, then Matlab should be used because JMP uses an imported Excel file as its input. Matlab has several resources to help users perform PCA. For this experiment, the "Analyze Quality of Life in U.S. Cities Using PCA" example was used to develop a Matlab script to perform PCA [30]. However, PCA methods for big data discussed in Section 2.4.2 should be used to formulate the Matlab script if the dataset is too large for the computer memory.

The first PCA analysis should include all variables that will influence fuel consumption. The only variables that should be discarded are the variables that do not influence engine operation such as latitude, longitude, and odometer reading. The output of the first PCA should be a biplot of the first two principal components with the variables labeled, as explained in "Analyze Quality of Life in U.S. Cities Using PCA [30].” This will show which variables are most influential because of the distance each variable's respective point is from the origin. Figure 4.3 shows an 
example of a biplot, where variables 3, 9, and 10 are the most influential to the variation in the dataset because they are the furthest away from the origin. However, these variables appear in different quadrants. Variable 3 has a positive correlation with the first and second principal components because it is located in the first quadrant. On the other hand, Variables 9 and 10 are located in the third quadrant, which means these variables have negative correlations with the first two principal components.

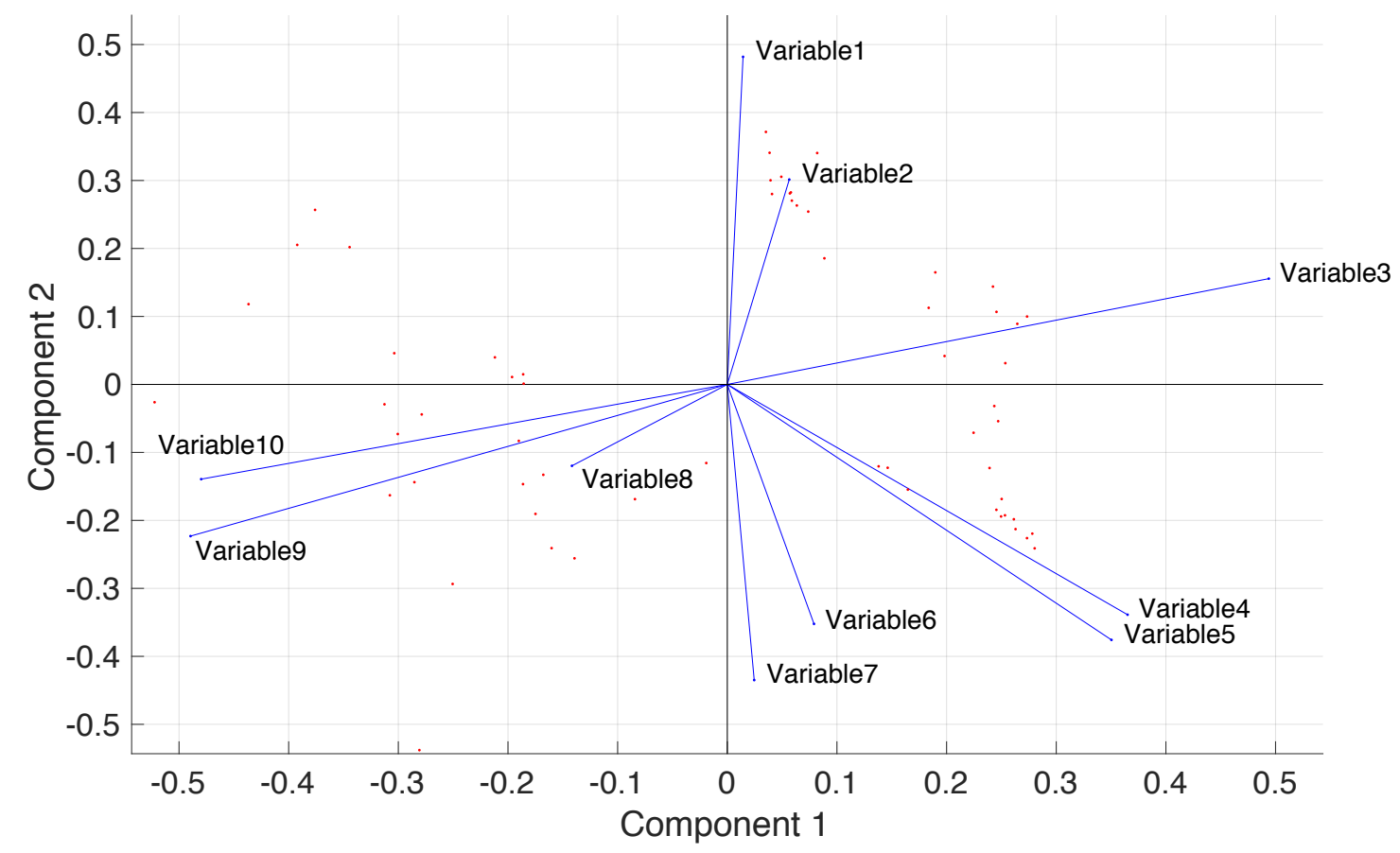

Figure 4.3: PCA Biplot Example

\subsection{Binning by Additional Variables}

In addition to separating the data based on the route, engine speed, and power, the data was separated by other variables using the PCA-analysis information, so that similar on-road operating conditions could be compared. A range of values should be selected for each variable along with a size for each bin. For example, the data can be separated by intake manifold temperature (IMT) 
between $10^{\circ} \mathrm{C}$ and $40^{\circ} \mathrm{C}$ in $5^{\circ} \mathrm{C}$ increments. Therefore, the first bin will contain the data from all variables where the IMT is between $10^{\circ} \mathrm{C}$ and $15^{\circ} \mathrm{C}$.

Each bin from the first variable bins can then be separated by another variable. Then, those bins can be separated by an additional variable and so on. The number of variables the data can be binned by is limited to the size of the data set. In this work, the data was binned by 3 additional variables. In order to draw a statistically significant conclusion, there should be at least 40 data points in the last bin to analyze [38]. Therefore, the variables and ranges selected for binning should be carefully determined based on the number of points in the raw data set. For example, if there are 1000 data points and separating the data into bins based on the IMT in $1{ }^{\circ} \mathrm{C}$ increments leaves 10 data points in each bin, then the increment should be increased to include more than 40 points in each bin.

The number of variables chosen for binning is limited depending on the total number of data points, as shown in Equation (15):

$$
x_{p}=y \prod_{i=1}^{m} N_{i}
$$

where $x_{p}$ is the total number of data points after data reduction, $N_{i}$ is the number of bins for variable $i, m$ is the number of independent variables, and $x_{p}$ is the minimum statistically-significant number of points per bin ( $y=40$ in this study). Therefore, if the number of independent variables $m$ can be determined based on $N_{i}$. Equation (16) shows the value of $m$ assuming the same number of bins $(N)$ for each variable:

$$
m=\frac{\ln \left(x_{p} / y\right)}{\ln (N)}
$$

As mentioned in Section 4.2, there were approximately 2-million usable data points per month per vehicle. Assuming an arbitrary number of 10 bins per variable and a minimum of 40 points in each 
bin, $m$ was calculated to be 4.699 . The actual value of $m$ must be rounded to the next lowest integer because the number of variables cannot be fractional and the number of points in each bin would be less than the minimum number of points in each bin. Therefore, the data can be binned by maximum 4 variables.

If the range of values for variable $i$ is small, the number of bins should also consider the accuracy of the measuring instrument. For example, the barometric pressure sensor used in the PAMS has an accuracy of \pm 170 pascals. Therefore, the range of each bin should not be less than 340 pascals to ensure the true value will lie within the bounds of each bin.

As the number of bins for each variable is arbitrary, Eq. 3 shows that a lower number of bins will increase $m$. This can be done using the results of the PCA analysis. The variables that are most influential on the variation in the dataset should have a higher number of bins compared to the variables that are less influential. This is because small changes in the most influential variables will influence the data more than small changes in the less influential variables.

The procedure for binning the data in this work was developed in a Matlab environment where for loops were used to cycle through each data point and if loops were used to assign a data point for each variable into a bin based on its value and the range of values for the variable used for binning. The number of bins must be selected rather than selecting the range for each bin because of Matlab limitations. Structures were used to organize the data into bins because each bin was easily accessible for analysis using dot-indexing. Figure 4.4 shows a visualization of the structures for data binned by two variables and 3 bins per variable. 


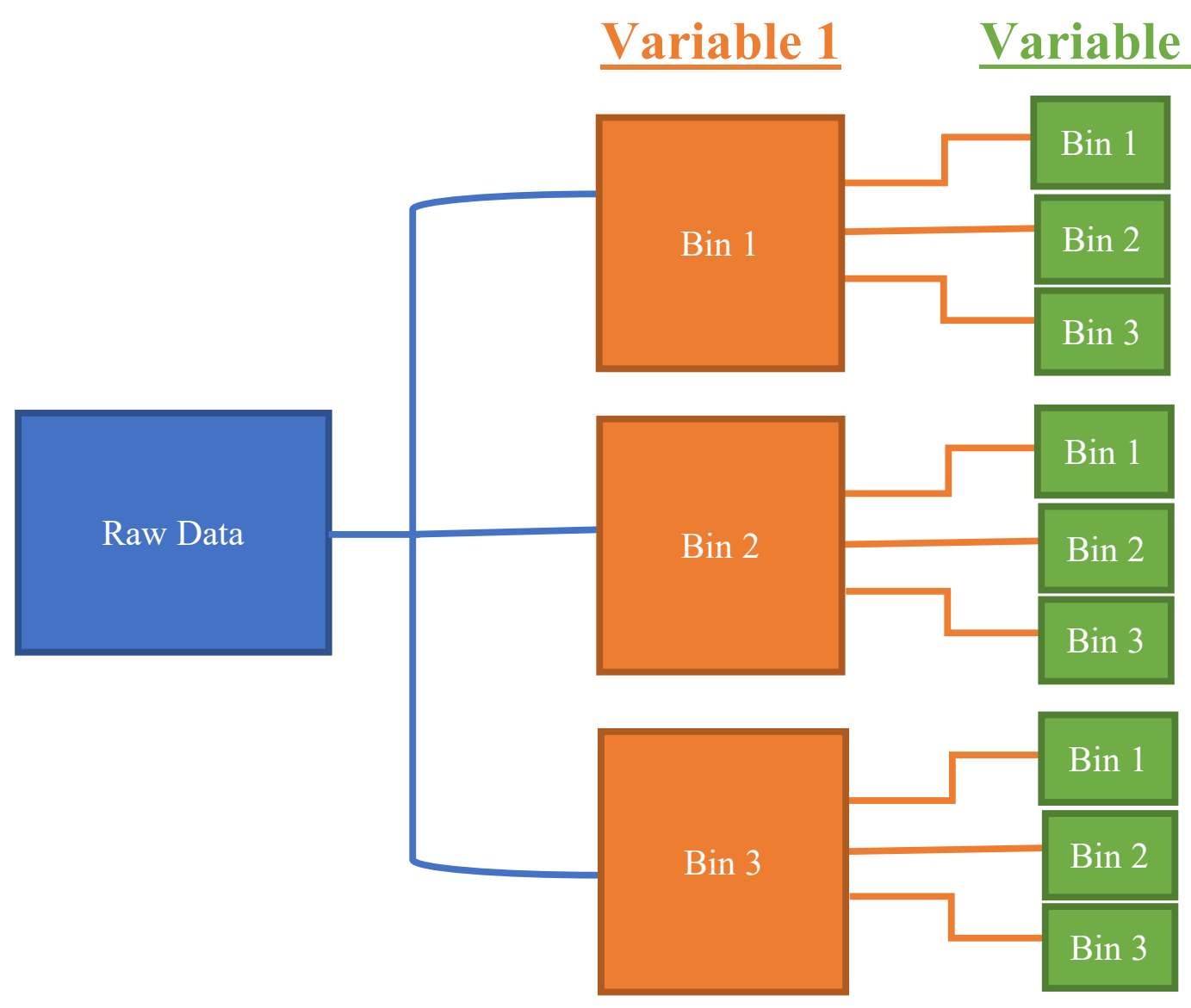

Figure 4.4: Visualization of Binned Matlab Structure

\subsection{Comparing Data Sets}

\subsubsection{Principal Component Analysis}

The PCA should be repeated with all variables included after the data has been separated into bins. This will reduce the variability of the most influential variables and allow the user to determine if small changes in those values greatly affect the data. There will be the same number of biplots as there are combinations of bins. If the distance between the origin and a variable's 
point is much smaller than other variables in the biplot, then those variables should be omitted from the next completion of PCA. This is because it indicates that changes in those variables are not influencing the variation of the dataset as much as the other variables. However, the variable of interest should always be included in the PCA, which in this case was the ECU's broadcasted fuel rate. This process should be repeated at the user's discretion until a desired number of influential variables is obtained.

For each completion of PCA, in addition to the biplot, the first 2 or 3 principal components should be plotted against each other for each dataset and for each vehicle. For example, comparing the first two or three principal components from each month of testing will show if there is a difference between datasets based on the variables included in the PCA. This is because the principal components are calculated based on the correlation between variables. Therefore, if the correlations are similar, the principal components will be similar. However, if there is a difference between the correlations, the principal components will be different and will show a separation between datasets. In other words, it will not define whether one dataset is better or worse than the other, but it will show if there is a difference that should be investigated further.

Ideally, if the fuel made a difference on the fuel consumption, the principal components for the two months where Fuel A was used would be clustered together, while the principal components for the month where Fuel B was used would be displayed as a separate cluster away from the other two clusters. An example of separation is shown as Figure 2.2.1 in Section 2.4.1. If there is no separation, then the principal components from each dataset will all be clustered together. Figure 4.5 shows an example of principal components that do not separate, where each color symbolizes a different dataset. 


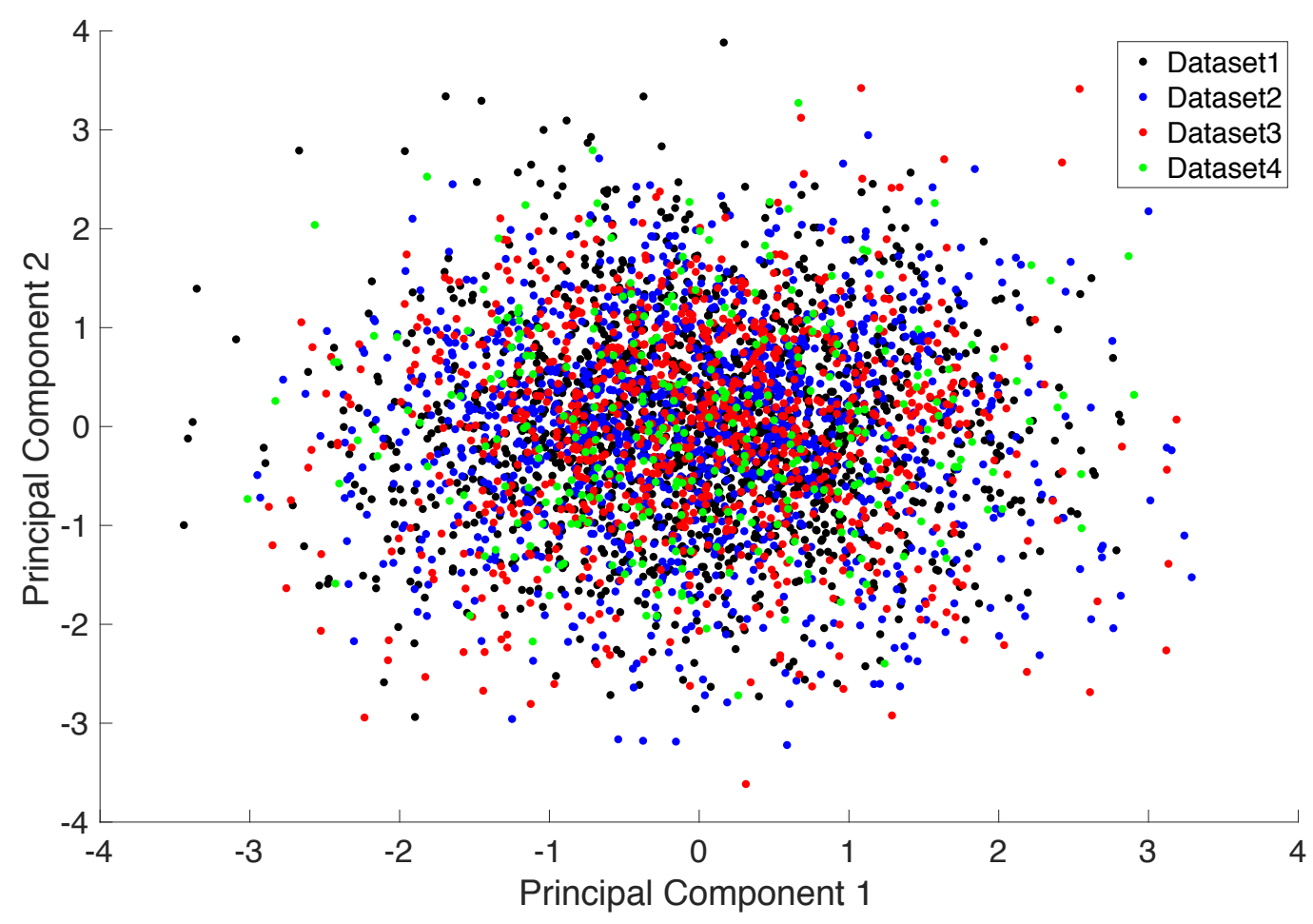

Figure 4.5: Principal Component Comparison Without Separation

The PCA completed for all combinations of bins should be repeated until there is separation between principal components for the different datasets. If the datasets do not completely separate using PCA, the difference between datasets may be too small for the PCA to capture.

\subsubsection{Percent Difference}

The percent difference method begins by calculating the averages and standard deviations of all variables for each bin in each data set. After these calculations are made, the averages of a variable from one dataset can be compared to another dataset. The percent difference between the average of one dataset and the average of a second dataset can be found using Equation (17): 


$$
\% \text { Difference }=\frac{\mu_{2}-\mu_{1}}{\mu_{1}} * 100 \%
$$

where $\mu_{1}$ and $\mu_{2}$ are the averages of the first and second datasets, respectively. In this case, the first dataset is the reference, so the percent difference will show whether or not the second dataset shows a larger or smaller value than the first dataset for a specified variable. The averages must only be compared if they are from the same bin in each dataset. Therefore, all bins that are not shared between datasets should be omitted.

There will be thousands of bins if the data is binned by several variables, especially if each bin's range is narrow. All these bins need to be compared to draw a conclusion, which can be very difficult without using a visual aid. After the percent differences for each bin between two datasets is calculated, a histogram should be generated to determine if there is a trend for the difference between datasets.

Figure 4.6 shows an example of a histogram of the percent differences of a variable for many bins, which shows that the second dataset's average values are higher than the first dataset's averages values for an arbitrary variable. This is because the distribution is skewed such that there are more bins that show a positive percent difference than a negative percent difference. On the other hand, Figure 4.7 shows the opposite scenario, where the second dataset's average values are smaller than the first dataset's average values for an arbitrary variable because there are more bins with a negative percent difference than a positive percent difference. Finally, Figure 4.8 shows a situation where there is no difference between the averages of each dataset for an arbitrary variable because the percent differences are normally distributed and are centered at zero. 


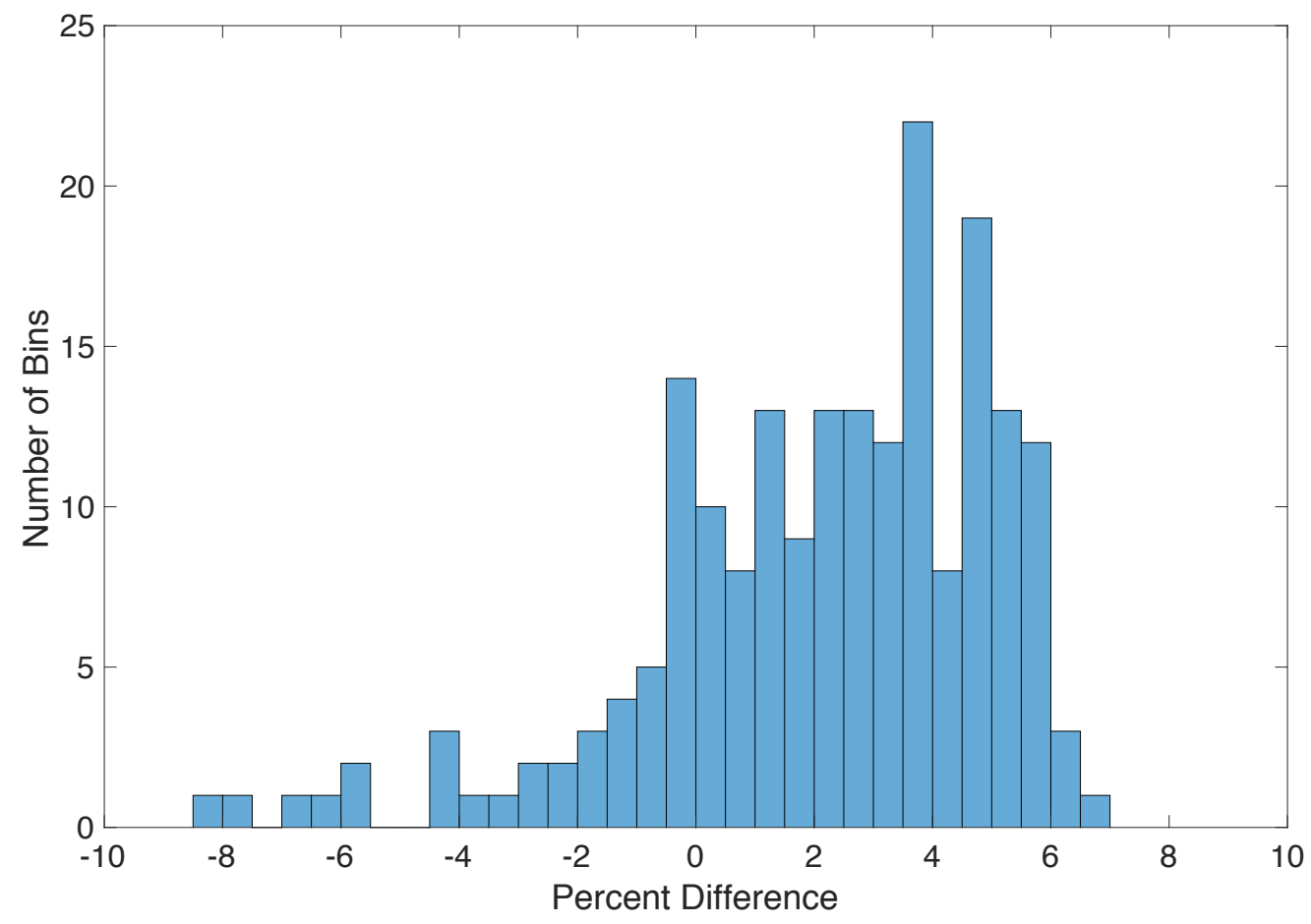

Figure 4.6: Positive Percent Difference Histogram Example

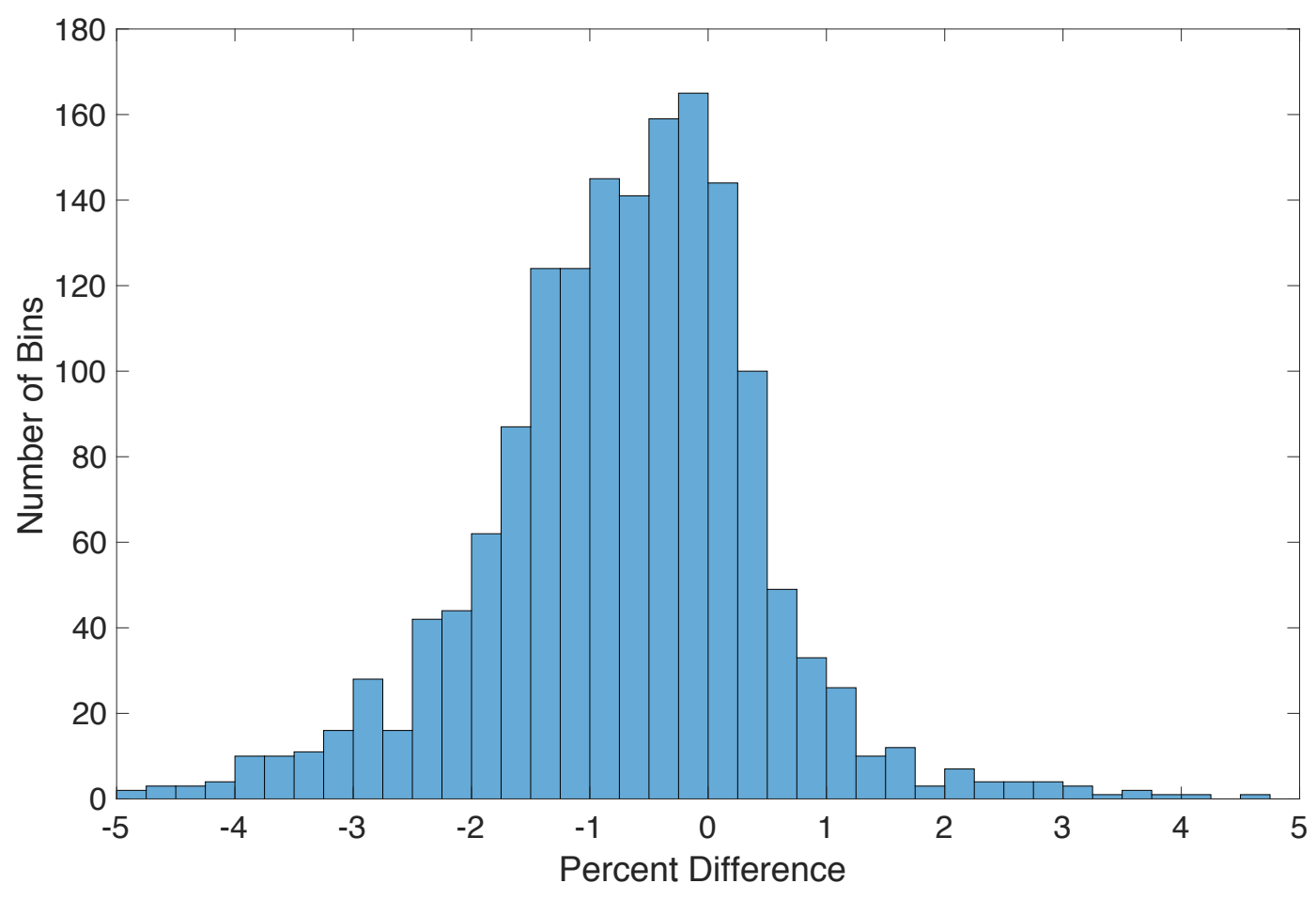

Figure 4.7: Negative Percent Difference Histogram Example 


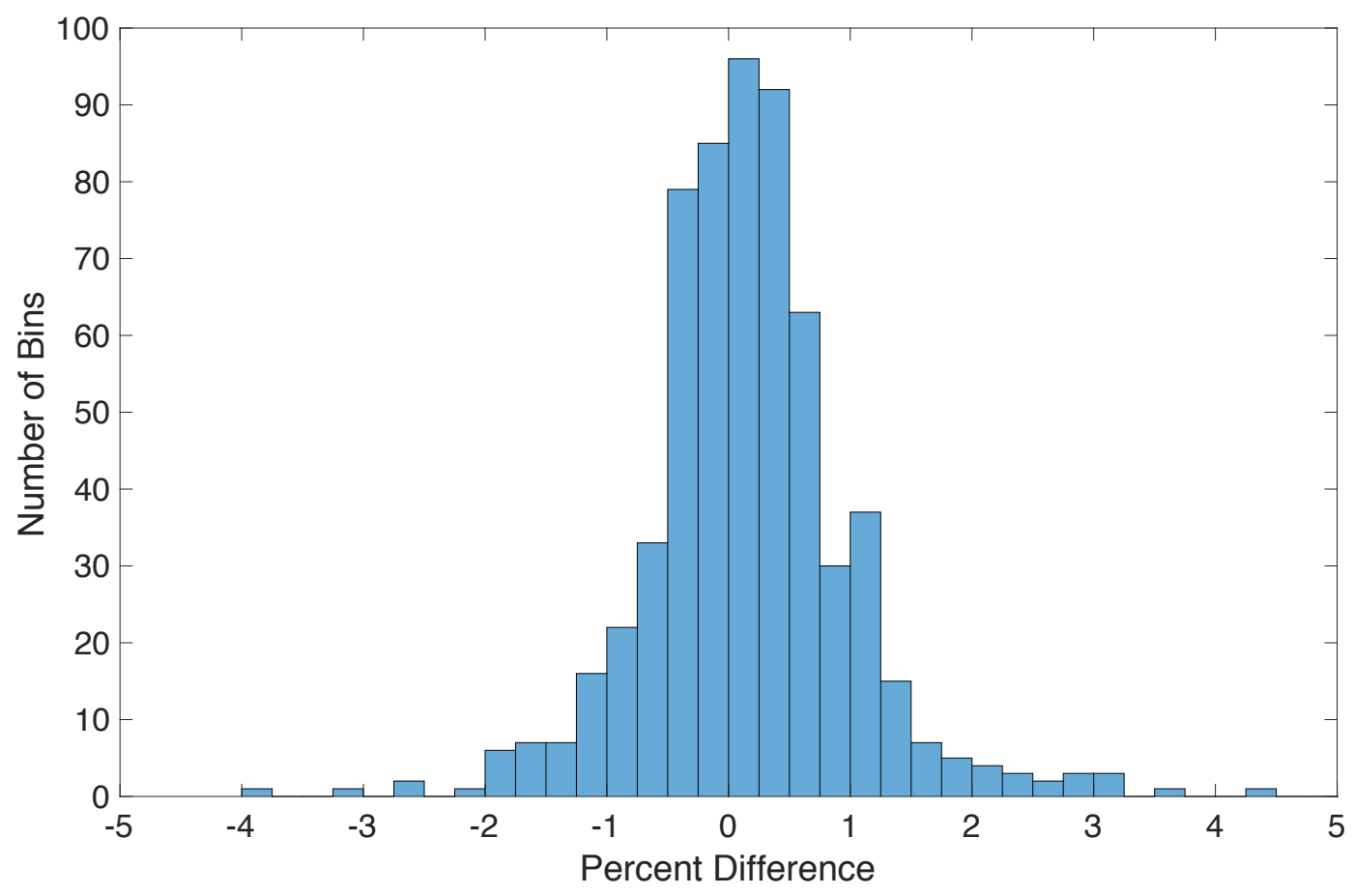

Figure 4.8: Zero Percent Difference Histogram Example

The number of percent difference values above and below zero should be counted as part of the analysis of the distribution. These counts are used to determine whether or not the difference between datasets is statistically significant. The percentage of values above and below zero should be calculated to determine the proportion of positive and negative percent differences for the distribution. Then, a normal model can be applied to determine statistical significance as long as the following conditions are met [38]:

1. The data has been selected at random

2. There must be at least 10 possible "successes" and "failures"

3. The sample size must be less than $10 \%$ of the population size 
To satisfy the first condition, the data should be collected such that the value each point is not dependent on other values. For this work, this condition was satisfied because each data point is independent of one another.

The ten possible "successes" and "failures" for the second condition are calculated based on the proportions that are considered a "success" or a "failure." For example, the successful proportion $(p)$ for fuel consumption data is the proportion of bins that showed a negative percent difference of the test fuel compared to the baseline fuel. This is because a negative percent difference infers lower fuel consumption for the test fuel. On the contrary, the failure proportion (q) for fuel consumption data is the proportion of bins that showed a positive percent difference of the test fuel compared to the baseline fuel. Therefore, in order to satisfy condition 2 the number "successes" and "failures" should be calculated using Equations (18) and (19):

$$
\begin{aligned}
& n \cdot p \geq 10 \\
& n \cdot q \geq 10
\end{aligned}
$$

where $n$ is the population size [38]. This means that should be at least ten possible successes and failures based on the success and failure proportions and the population size.

Finally, to fully satisfy the third condition, the data from each bin should be randomly sampled and the sample should be used to calculate the mean of each bin. The sample should be less than $10 \%$ of the population [38]. However, in this work, the entirety of each bin was used to calculate the mean because these were considered as samples from all possible data points that could be collected.

The percentage of bins with positive and negative percent differences should be calculated if the three conditions are met and the normal model can be applied. The proportion that is higher should be used to determine if the difference between datasets is statistically significant. This proportion is referred to as $P$. This proportion should be compared to the proportions of a perfectly 
normal distribution that shows no difference between datasets. In this case, a perfectly normal distribution would have $50 \%$ of the points below zero and $50 \%$ above zero. Therefore, $p$ and $q$ would be equal to 0.5 . The standard deviation (SD) of a normal distribution of proportions can be found using Equation (20) [38]:

$$
\mathrm{SD}=\sqrt{\frac{p q}{n}}
$$

Then, the z-score can be calculated using Equation (21) [38]:

$$
\mathrm{z}=\frac{P-p}{\mathrm{SD}}
$$

The z-score can then be used to determine the P-value, which determines statistical significance based on a user-determined limit. In this work, the P-value limit was 0.05. In other words, a conclusion was considered statistically significant if the P-value was less than 0.05 . The P-value can be determined using a normal distribution calculator, where the mean and standard deviations are set at 0 and 1 , respectively. The P-value is the probability that the actual value is above the $\mathrm{z}-$ score.

\subsection{Binning Based on Shape of Distribution}

If the binning and comparison techniques described in Sections 4.7 and 4.8 do not show a difference between datasets, there may not be a difference, or other techniques should be employed to separate the data. The data from each vehicle for each segment of testing should be binned first by route, engine power and engine speed. The ranges of power and engine speed should include the full range of possible values for these variables.

\subsubsection{Binning Assuming a Normal Distribution}


After binning by route, power, and engine speed, the data should be separated based on additional variables chosen based on the PCA, and the range of values available for binning should be based on a $95 \%$ confidence interval assuming a normal distribution. This means that the mean and standard deviation of each desired variable should be calculated for each bin. This method will prevent outliers from influencing the data analysis.

The lower and upper bounds for the range of values for each desired variable should be found by subtracting and adding two standard deviations to the mean, respectively. These values should be rounded to the nearest integer or multiple of ten for simplicity. However, each segment of testing could potentially result in different ranges for a given variable. Therefore, the largest lower bound and the smallest upper bound should be selected as the common range for all segments for each vehicle.

After the range is determined, it should be segmented based on the desired number of bins. Next, the data should be binned based on this range for the desired variable. As an example, the first desired variable is the IMT. For one bin for an arbitrary combination of route, engine power and engine speed, the average IMT was $30^{\circ} \mathrm{C}$ and the standard deviation was $10^{\circ} \mathrm{C}$. Then, the $95 \%$ confidence interval range of values for the IMT would be $10^{\circ} \mathrm{C}$ to $50^{\circ} \mathrm{C}$. If the number of bins desired is ten, then the bins for the IMT would each contain a range of $4^{\circ} \mathrm{C}$. Then, all variables should be separated into these bins based on the values of IMT. The process should be repeated for each additional desired variable.

\subsubsection{Binning Accounting for Shape of Distribution}


This method for determining the range for a desired variable can be used as a starting point. However, the range may not be optimal if a significant number of data points was lost during the binning process. Taking into account the shape of each desired variable's distribution, rather than assuming a normal distribution, can help optimize the range for each chosen variable for binning. But, plotting a histogram for each bin combination and determining its shape is not feasible when there are thousands of bin combinations. There are two factors, however, that provide a numerical value for the shape of the distribution: kurtosis and skewness.

The kurtosis of a distribution is the sharpness of its peak. For example, the kurtosis of the distribution in Figure 4.9 (3.35) is lower than the kurtosis of the distribution in Figure 4.10 (580). Therefore, the kurtosis of a distribution can be used to identify uniform or very narrow distributions. For this work, Matlab's kurtosis function was used to determine the kurtosis of the distribution of a desired variable for each bin combination. For this function, a value of 3 indicates a perfectly normal distribution. A value less than 3 indicates a more uniform than normal distribution, while a value greater than 3 indicates a narrower than normal distribution.

The skewness of a distribution determines how far the center of the distribution strays from the center of the range of values in the distribution. Matlab's skewness function was used in this work to identify the skewness of the distribution of each bin. The skewness function returns a value of zero if the distribution is perfectly centered. However, the value is negative if the data is concentrated to the right of the center and the value is positive if the data is concentrated to the left of the center. For example, the skewness of Figure 4.9 is negative (-0.63), while the skewness of Figure 4.10 is positive (15.97). 


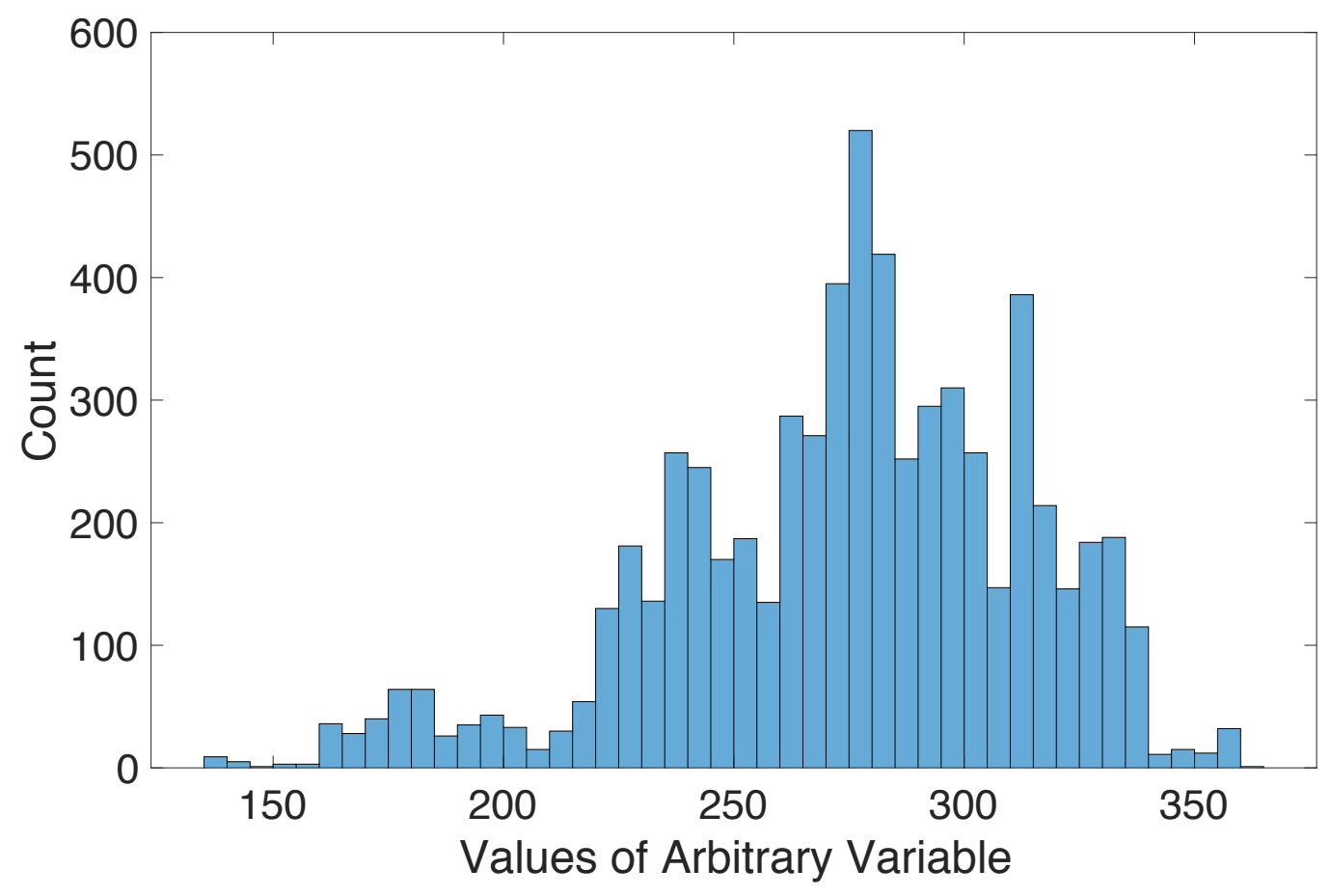

Figure 4.9: Low Kurtosis and Negative Skewness Example

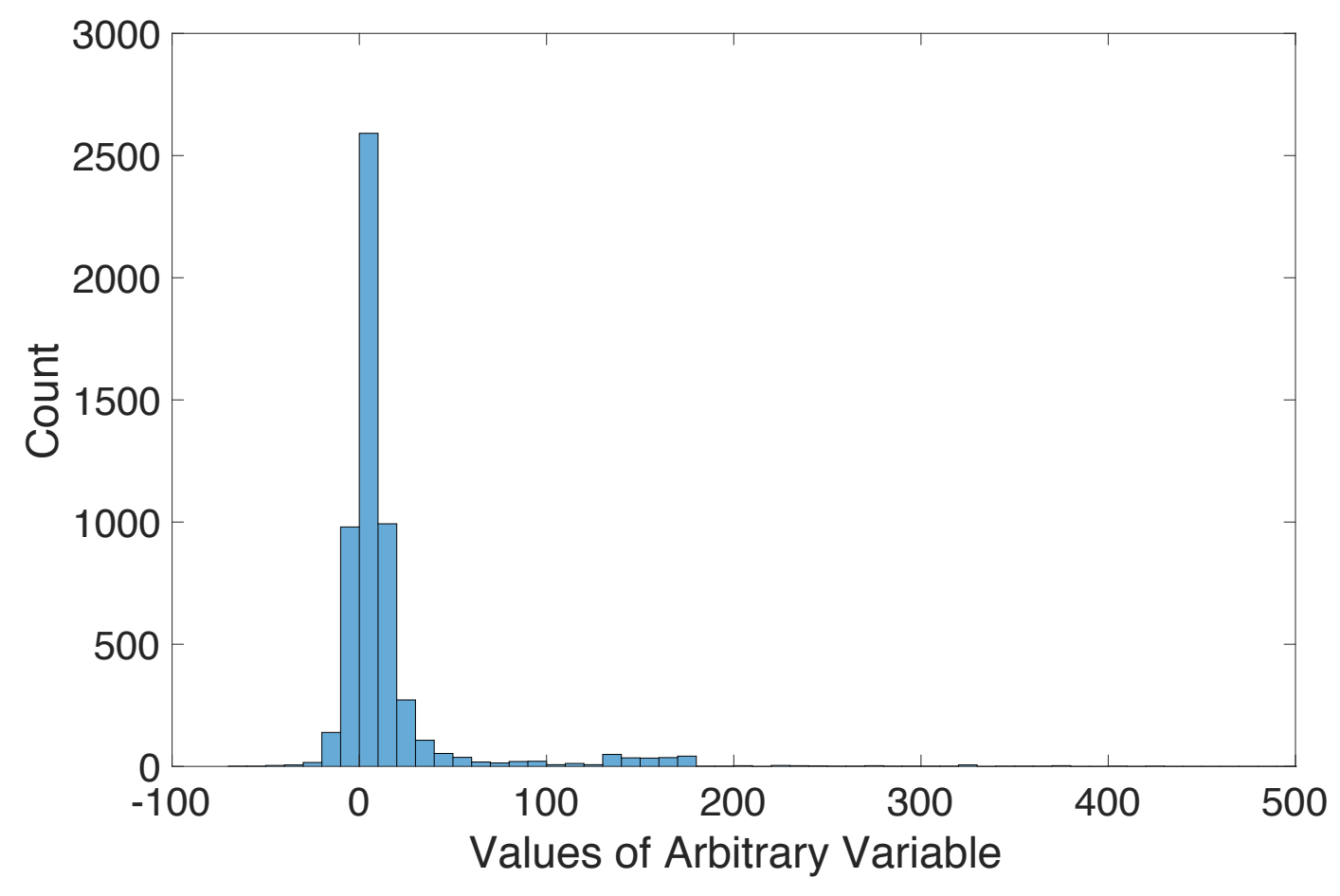

Figure 4.10: High Kurtosis and Positive Skewness Example 
Therefore, the kurtosis and skewness values can be used to optimize the binning range for each desired variable since these values describe the shape of the distributions, hence the number of bins for variable $i$ can decrease. For example, after the data was binned by route, engine power and engine speed, the kurtosis and skewness of the distribution for the first desired variable in each bin combination was calculated. The range was determined using a $95 \%$ confidence interval, as previously discussed, if the kurtosis and skewness values indicated a nearly normal and centered distribution. However, assuming a nearly centered distribution, if the kurtosis value was much lower than 3 , the range should be widened because the distribution is more uniformly distributed across the range of possible values. For example, the range can be widened such that the lower and upper bounds of the range are three standard deviations from the mean if the kurtosis value is 1 . On the other hand, the range can be narrowed if the kurtosis value is greater than 3 because the points are concentrated in one area. For example, the range can be narrowed such that the lower and upper bounds of the range are only one standard deviation from the mean if the kurtosis value is 5 .

However, the mean is not representative as the center of a distribution if the distribution is skewed. The median should be used as the center of the distribution if it is skewed in either direction [38]. Therefore, the skewness value can be used to determine if the median should be used as the center of the range instead of the mean. If the magnitude of the skewness value is approximately zero, the mean should be used as the center of the range. On the other hand, the median should be used as the center of the range if the magnitude of the skewness value is larger than zero.

The skewness of a distribution can also be used to optimize the range. If the magnitude of the skewness value is larger than zero, then the range can be determined such that the upper and lower bounds are not the same distance away from the median. Figure 4.11 shows an example of a 
distribution, where the properties of the distribution can be found in Table 4.1. The median should be considered as the center of this distribution since the skewness value is greater than zero. Then, the upper bound should be closer to the median than the lower bound because the data is more concentrated on the right side of the median than the left side of the median. The exact distances can be determined at the user's discretion, but the total range of values should be considered. For example, in Figure 4.11, the upper bound of the range was selected as one standard deviation away from the median because that value captures all the data points above the median, where there was a high concentration of data points. The lower range was selected as two standard deviations away from the median because the kurtosis value was larger than 3 , so it is known that most of the data is concentrated around the median. However, because the distribution is skewed, two standard deviations from the median ensures that most of the data is captured, while removing outliers that are far away from the median. 


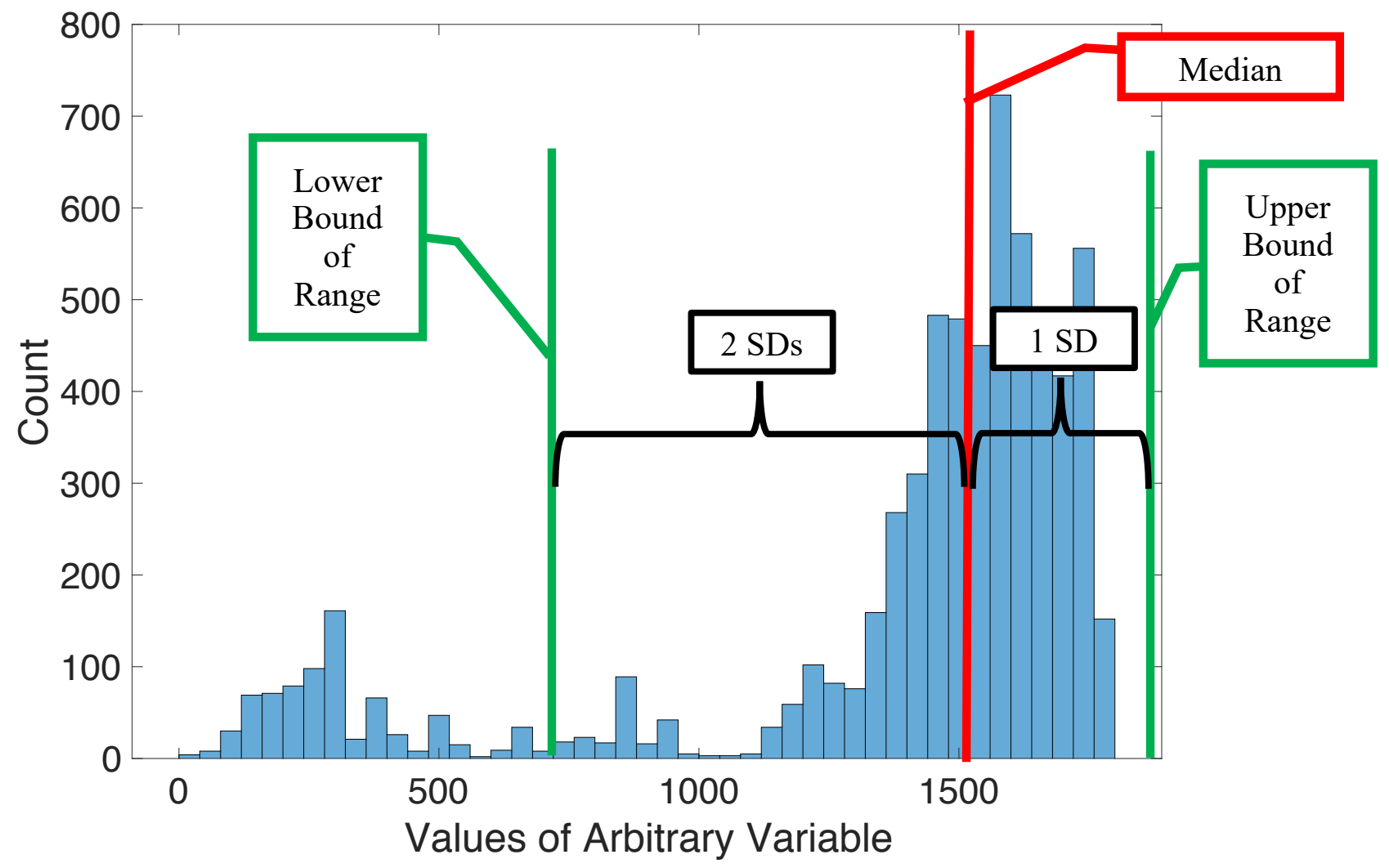

Figure 4.11: Example of Range Optimization for Skewness

Table 4.1: Properties of the Distribution in Figure 4.11

\begin{tabular}{|c|c|}
\hline Mean & 1382 \\
\hline Median & 1536 \\
\hline Standard Deviation & 437 \\
\hline Kurtosis & 4.99 \\
\hline Skewness & -1.79 \\
\hline
\end{tabular}

It is important to remember that a common range must be established for each segment of testing in order to compare bins between route segments. Therefore, for each desired variable for binning, the mean, median, standard deviation, kurtosis, and skewness values should be calculated 
for each test segment. The averages of these values should be calculated for each vehicle to obtain representative values for all test segments for each vehicle. These values can also be rounded for simplicity. Then, the range can be determined based on the average kurtosis and skewness values. After the range is selected, a number of bins should be selected, and the width of each bin can be calculated. It is important to remember that the size of the bin should not be smaller than the accuracy of the measurement device as discussed in Section 4.7. Then, the data from all the other variables can be placed into these bins based on the value of the desired variable, exactly as it was explained at the end of Section 4.9.1. This process can be repeated for as many variables as possible, as calculated using the equations in Section 4.7.

After the binning is completed for each segment of testing and each vehicle, the bins should be compared using the percent differences of the averages between bins from different test segments as described in Section 4.8.2. 


\section{Summary, Conclusions, and Recommendations for Future Work}

\subsection{Summary and Conclusions}

This study described a method to determine fuel consumption differences between two fuels. Three vehicles (two test vehicles and one control vehicle) were operated over a period of three months. The test vehicles used the baseline fuel for the first and third months and used the test fuel during the second month. The control truck used the baseline fuel for the duration of testing. Positive-displacement flow meters (KRAL Model OME13 Compact) were used to measure the actual fuel consumption and compare it with the one estimated by the ECU. A torque meter (Accumetrics Model AT4500) measured the driveshaft torque. A portal activity monitoring system (PAMS) gathered and stored GPS and ambient conditions data. The PAMS also stored the ECU, flow meter, and torque meter data.

The data was binned based on variables that had the most influence on the data, which were determined using principal component analysis (PCA), and the average ECU fuel rate was compared for each bin for each fuel. The bins were compared by calculating the percent difference between the average fuel rate of each bin, for all data points in a particular period of time or based on routes. The results were tested for statistical significance using the proportions of bins above and below zero and comparing to proportions of a perfectly centered distribution. The z-scores and respective P-values were calculated, and the results were considered statistically significant if the P-value was below 0.05 . The exact results could not be revealed here due to the proprietary nature of the testing. However, this study described the methodology used to collect and analyze the data.

The main conclusions from this study were: 
- Each month of testing provided approximately 4.5-million data points per vehicle, recorded at a frequency of $10 \mathrm{~Hz}$. Data reduction significantly decreased the total number of data points to approximately 2 -million per month and per vehicle (i.e., $55 \%$ of recorded points were discarded). Specifically, the analysis removed engine cutoff points, idling points, and data from days where it rained or snowed. As less than $1 \%$ of the possible bins contained at least 40 data points for any route, more testing should have been conducted to gather more data points.

- This study was conducted from October 2018 through January 2019. Therefore, there were significant temperature differences over the months of testing. This proved to be a major cause of reducing available bins for comparison because the ambient temperature was not comparable from month-to-month.

- The routes had an effect on the number of bins available for analysis. The routes with many hills had the fewest number of bins available for analysis due to the removal of the engine cutoff points. The route that provided $95 \%$ highway data (Route 2 in Table 3.4) allowed the greatest number of bins with more than 3,000 bins available for comparison. However, most of these bins originated from the segment of the route between Morgantown, WV and Clarksburg, WV, where the hills are not as steep as the rest of the route.

- A large number of bins provides a large number of engine operating conditions available for comparison, so it is more likely that a statistically significant conclusion can be drawn from the data.

- PCA identified the variables that have the most influence on the variation in a dataset. It also helped to find the variables that have little influence on the variation, so these variables could be neglected in the binning process. PCA was also useful for determining if there was a difference between datasets based on the variables included in the analysis. 
- Since fuel consumption is dependent on many variables, binning the data by the most influential variables reduced the variation in fuel consumption between the data points inside a bin. Comparing these bins between segments of testing allowed for statisticallysignificant conclusions to be drawn.

- The kurtosis and skewness indicated the shape of the data points distribution inside a bin without having to plot a histogram. These parameters are useful for determining the range of values for a variable that is to be used for binning. However, the kurtosis and skewness values can be assumed for certain engine operation parameters. For example, for data binned by route, it can be assumed that the vehicle speed for any given route will have a kurtosis value greater than 3 . This is because there will not be a lot of variation in speed for a given route, so the vehicle speed distribution will be narrower than normal. On the other hand, it can be assumed that the distribution of ambient temperature has a kurtosis value less than 3 . This is because the ambient temperature will fluctuate throughout testing and will have a wider than normal distribution. The skewness values of both of these parameters can be assumed to be zero.

- The flow meters could not be used for instantaneous fuel consumption values because the time alignment between the ECU command and the flow meter reading varied with many parameters such as fuel temperature, fuel pressure inside the multiple delivery and return lines, fuel filter characteristics, engine operating conditions, etc. Therefore, the data from the flow meters was integrated over longer period of times then compared to the ECU values. As the average percent difference of the daily fuel consumption between the ECU and flow meter was approximately $2.5 \%$, the flow meter data confirmed that the ECU data was accurate. 
- The preliminary testing for the flow meters showed a $0.04 \%$ difference between the fuel consumption according to flow meters and the ECU for a single FTP engine cycle. More FTP cycles should have been completed to confirm this low percent difference value.

- The flow meters were sensitive to debris inside fuel lines that may prevent the rotation of the internal screws used to calculate the volumetric flow rate of the fuel. If the screws did not rotate, the fuel was still able to travel through the flow meter, but the volumetric flow rate showed a value of zero. Therefore, a mesh filter should be used to remove any debris prior to entering the flow meters.

- The torque meter added additional insight to the actual engine output torque as the torque is not typically measured or broadcasted by the ECU. However, it was extremely sensitive to excessive vibration experienced during on-road testing. There were instances when excessive vibration resulted in the failure of the brass ring and electrical connections between the strain gage and transmitter. However, on some occasions, the receiver indicated proper data collection, but the failed electrical connections between the strain gage and transmitter produced unusable data. Therefore, the data from the torque meter had to be checked daily to ensure proper operation.

- Even though the transmitter was mounted as closely as possible to the transmission end of the driveshaft to minimize lateral movement, some lateral movement was inevitable. This movement caused the transmitter to occasionally come into contact with the ring, which caused failure of the strap that held the transmitter around the driveshaft. Since the strap is the only mechanism holding the transmitter to the driveshaft, the centrifugal forces will eject the transmitter from the driveshaft if the strap fails. Therefore, the condition of the strap had to be monitored daily to determine if it was deteriorating. 
- The strain gage for the torque meter withstood the excessive vibration and all ambient conditions for the duration of testing using the fiberglass-cloth installation procedure with the AE-10 adhesive discussed in Section 3.6.4.2. Therefore, this strain gage installation method is suitable for on-road testing.

\subsection{Recommendations for Future Work}

This study suggested that while stringent requirements should be imposed to limit data variability, testing should be completed for a longer period of time and include more fuel switching. Longer testing will also provide points for a larger range of ambient temperatures, which will allow for more bins available for comparison between test segments. Testing should also occur in a dry climate to avoid losing data points because of rain. Additionally, if on-road testing is necessary, the routes selected for testing should avoid grade changes to avoid losing data points due to engine cutoff.

In future works, it is recommended to use the exact same make, model, and model year vehicles for all vehicles. In this work, the control vehicle was newer than the test vehicles, but was the same make and model, so it was deemed acceptable. However, the control vehicle's results were not consistent with the test vehicles' results when data analysis was completed. It could not be determined if the differences were due to the control vehicle not being the same model year and/or its engine having a higher power rating than the test vehicles. Therefore, the control vehicle could have had differences in operation that could not be compared to the test vehicles.

The results recommended the use of flow meters for future work. This particular flow meter model functioned reliably with little maintenance and it was easily integrated into the data acquisition system. The additional fuel meter display was very useful because it allowed the user to observe the data being collected in real time from both flow meters. If using a filter is not used 
as discussed in the previous section, the flow meter activity should be checked daily to ensure the screws are rotating. The screws should be removed and cleaned with a lubricating detergent, such as WD-40, if the screws are not turning.

The particular torque meter used in this study is not recommended for on-road data collection because of its sensitivity to vibration. However, if it is the only option, the condition of the strap should be closely monitored, and if there is evidence that the strap has been rubbing on the brass ring, a new strap should be ordered and adjustment of the placement of the ring should be considered. Also, the brass ring should be mounted in a way such that the ring can move laterally with the driveshaft to avoid any contact with the transmitter.

During analysis of the torque meter data, it was realized that any trends observed in the data could not be validated because the strain gage calibration was only completed at the beginning of testing. Consequently, it could not be determined whether or not the strain gage signal changed or remained the same for similar torque values throughout testing. It is recommended that the strain gage calibration is completed periodically throughout testing in order to have confidence in any trends that may be observed. 


\section{References}

[1] J. Visser, T. Nemoto, and M. Browne, "Home Delivery and the Impacts on Urban Freight Transport: A Review," Procedia - Social and Behavioral Sciences, vol. 125, pp. 15-27, 2014.

[2] E. Hellstrom, M. Ivarsson, J. Aslund, and L. Nielsen, "Look-ahead control for heavy trucks to minimize trip time and fuel consumption," Control Engineering Practice, vol. 17, pp. 245-254, 2009.

[3] G. Fontaras, N.-G. Zacharof, and B. Ciufo, "Fuel consumption and CO2 emissions from passenger cars in Europe-Laboratory versus real-world emissions," Progress in Energy and Combustion Science, vol. 60, pp. 97-131, 2017.

[4] E. P. Agency. "Sources of Greenhouse Gas Emissions." https://www.epa.gov/ghgemissions/sources-greenhouse-gas-emissions (accessed April 5, 2019).

[5] M. I. Piecyk and A. C. McKinnon, "Forecasting the carbon footprint of road freight transport in 2020," INt. J. Production Economics, vol. 128, pp. 31-42, 2010.

[6] R. L. McCormich, L. B. A. Ryan, T. L. Daniels, J. yanoWitz, and M. S. Graboski, "Comparison of Chasis Dynamometer In-Use Emissions with Engine Dynamometer FTP Emisions for Three Heavy-Duty Diesel Vehicles," presented at the International Fall Fuels and Lubricants Meeting and Exposition, San Francisco, California, 1998.

[7] A. A. Zulkifli et al., "Impact of the electric compressor for automotive air conditioning system on fuel consumption and performance analysis," presented at the IOP Conference Series: Materials Science and Engineering, 2015.

[8] P. Oberg, P. Nyberg, and L. Nielsen, "New Chassis Dynamometer Laboratory for Vehicle Research," SAE International Journal of Passenger Cars - Electronic and Electrical Systems, vol. 6, no. 1, pp. 152-161, 2013.

[9] R. Carlson, H. Lohse-Busch, J. Diez, and J. Gibbs, "The Measured Impact of Vehicle Mass on Road Load Forces and Energy Consumption for a BEV, HEV, and ICE Vehicle," SAE International Journal of Alternative Powertrains, vol. 2, no. 1, pp. 105114, 2013.

[10] H. Y. Tong, W. T. Hung, and C. S. Cheung, "On-Road Motor Vehicle Emissions and Fuel Consumption in Urban Driving Conditions," Journal of the Air \& Waste Management Association, vol. 50, no. 4, pp. 543-554, 2000.

[11] C. J. Brace, R. Burke, and J. Moffa, "Increasing Accuracy and Repeatability of Fuel Consumption Measurement in Chassis Dynamometer Testing," Proceedings of the Institution of Mechanical Engineers, vol. 223, D, pp. 1163-1177, 2009.

[12] S. o. A. Engineers, "Surface Vehicle Recommended Practice J1321," in Fuel Consumption Test Procedure - Type II, ed, 2012.

[13] R. Burke, E. Chappell, K. Burke, M. Gee, and R. Williams, "Factors Affecting Test Precision in Latest Vehicle Technologies," presented at the WCX World Congress Experience, 2018.

[14] K. Ahn, H. Rakha, A. Trani, and M. Van Aerde, "Estimating Vehicle Fuel Consumption and Emissions based on Instantaneous Speed and Acceleration Levels," Journal of Transportation Engineering, vol. 128, no. 2, pp. 182-190, 2002.

[15] K. Boriboonsomsin and M. Barth, "Impacts of Road Grade on Fuel Consumption and Carbon Dioxide Emissions Evidenced by Use of Advanced Navigation Systems," Transportation Research Record: Journal of the Transportation Research Board, vol. 2139, pp. 21-30, 2009. 
[16] H. C. Frey, K. Zhang, and N. M. Rouphail, "Fuel Use and Emissions Comparisons for Alternative Routes, Time of Day, Road Grade, and Vehicles Based on In-Use Measurements," Environmental Science \& Technology, vol. 42, no. 7, pp. 2483-2489, 2008.

[17] R. Burke and C. Brace, "The Effects of Engine Thermal Conditions on Performance, Emissions and Fuel Consumption," presented at the SAE 2010 World Congress \& Exhibition, 2010.

[18] M. Ivarsson, J. Aslund, and L. Nielsen, "Impacts of AMT Gear-Shifting on Fuel Optimal Look Ahead Control," presented at the SAE 2010 World Congress and Exhibition, 2010.

[19] J. J. Eckert et al., "Fuel Consumption Reduction Based on the Optimization of the Vehicle Gear Shifting Strategy Considering New Gear Ratios," presented at the 24th SAE BRASIL International Congress and Display, Sao Paulo, Brasil, 2015.

[20] T. Lee and J. Son, "Relationships between Driving Style and Fuel Consumption in Highway Driving," presented at the 16th Asia Pacific Automotive Engineering Conference, 2011.

[21] C. Wang, M.-H. Chen, E. Schifano, J. Wu, and J. Yan, "Statistical Methods and Computing for Big Data," Statistics and Its Interface, vol. 9, no. 4, pp. 399-414, 2016.

[22] RiskAMP, "What is Monte Carlo Simulation?,"

[23] S. Kullback and R. A. Leibler, "On Information and Sufficiency," The Annals of Mathematical Statistics, vol. 22, no. 1, pp. 79-86, 1951.

[24] N. Lin and R. Xi, "Aggregated Estimating Equation Estimation," Statistics and Its Interface, vol. 4, pp. 73-83, 2011.

[25] E. D. Schifano, J. Wu, C. Wang, J. Yan, and M.-H. Chen, "Online Updating of Statistical Inference in the Big Data Setting," Technometrics, vol. 58, no. 3, pp. 393-403, 2016.

[26] T. Zhang and B. Yang, "Big Data Dimension Reduction using PCA," presented at the 2016 IEEE International Conference on Smart Cloud, New York, NY, 2016. [Online]. Available: https://ieeexplore.ieee.org/document/7796166/.

[27] H. Abdi and L. J. Williams, "Principal Component Analysis," Wiley Interdisciplinary Reviews: Computational Statistics, vol. 2, no. 4, pp. 433-459, 2010.

[28] P. Wagner, "Principal Component Analysis and Linear Discriminant Analysis with GNU Octave," in Bytefish.de vol. 2019, ed, 2011.

[29] G. Strang, Introduction to Linear Algebra, 5 ed. Wellesley-Cambridge Press, 2016.

[30] MathWorks. "Analyze Quality of Life in U.S. Cities Using PCA." (accessed February 25, 2019).

[31] E. Ericsson, "Independent Driving Pattern Factors and Their Influence on Fuel-Use and Exhaust Emission Factors," Transportation Research Part D, vol. 6, pp. 325-345, 2001.

[36] M. Measurements, "Strain Gage Thermal Output and Gage Factor Variation with Temperature," ed, 2014.

[37] N. Instruments, "Strain-Gauge Measurement - A Tutorial," ed, 1998.

[38] R. De Veaux, P. F. Velleman, and D. E. Bock, Intro Stats, 4th ed. Pearson Education, Inc., 2014. 